Logic \& Logical Philosophy

Jerzy Perzanowski

\title{
LOCATIVE ONTOLOGY
}

Parts I - III

To the memory of Wtadystaw Kania

(1924-1992)

Received February 26, 1992 


\section{CONTENTS}

Part I. Background

1. Introduction

2. Verb-Type-Ontologies

3. Verb-Type-Ontologics

4. Rudimentary Definitions

5. Preorders

6. Premereologies

Mereological Condensation

Overlapping

Condensation Revisited

Augmentation

The Scope of ME

Towards Mereology

Summary

7. Leśniewski's Ontology

Part II. Location

8. Basic Concepts, Kinds and Properties of Location The Idea

Fundamental Concepts of Location

Preliminary Observations

Transitivity Laws

9. Locative Ontologies

Axioms

Immediate Consequences of Axioms

Duality

Axioms Revisited

Preservation

Part III. Locative Onto-Logic

10. Preorders and Locative Structures

Generalia

Comparison

Comparison Continued

Mereolocation

Conclusion

(To be continued)

Acknowledgments and References 


\section{PART I. BACKGROUND}

\section{INTRODUCTION}

1.1 Being is said and interpreted in various ways. These are studied in ontology. Ontology is concerned with both particular beings of several sorts as well as with the being and Being itself - respectively beings' collection and unification into one ${ }^{1}$.

1.2 Ontology is the theory of what there is, and of why and how. It can be either descriptive (phenomenological), putting emphasis on the first component of the ontological question "what there is", or theoretical (speculative), trying to outline a logical view of its universe - the ontological universe.

Following Leibniz this space is here understood as the space of all possibilities, for to the second part of the ontological question "why and how?" we are looking for an answer of the form " $x$ is because $x$ is possible and, in addition, enjoys additional specific conditions".

1.3 Three closely related ontological notions are basic: the notion of a being, the notion of the being and the notion of Being. These are, to be sure, obscure and complex, covering under three expressions a rich variety of connected ideas.

They can be approached in at least three ways ${ }^{2}$ connectional or qualitative, and through what we shall call verb-type or relational ontologies.

1.4 The possibilistic approach is determined by Leibniz's question "How (a given) is possible?". A being is defined here as any possible object. In consequence, ontology equals the general theory of possibility.

\footnotetext{
${ }^{1}$ Cf. [15]

${ }^{2}$ For an extended modern discussion of all three approaches cf. [13], [15] and [23]. The possibilistic approach is discussed also in [14], [19], [21] and [22]; the qualitative one in [16] and [17],
} 
The qualitative, or connectional, approach develops the very traditional idea: a being is any subject of some qualities. The ontology here is confined to the theory of qualities, of subjects and of the connections between them.

The verb-type approach starts with an obvious observation that basic ontological notions are nominal derivatives of the verb "to be". Hence it consists in a clarification of the nominalizations of the verb "to be". Because of an influential traditional reducing of all affirmative statements to sentences of the form " $S$ is $P$ ", verb-type-ontology is dominated by its attributive or predicative variants.

1.5 Locative ontology, which I am going to discuss here, is a variant of verb-type-ontology determined by locative uses of the verb "to be", like: I am here. You are at home. She is in Schaan. But also I am in trouble (in writing this essay), etc.

It has been observed ${ }^{3}$ that locative uses are among the most primitive forms of the verb "to be" in Indo-European languages. On the other hand, they played a crucial role in the development of certain basic ontological concepts of ancient Greek philosophy ${ }^{4}$.

Notice next, that it is very unnatural to impose upon locative sentences the canonical form " $S$ is $P$ ". To this end people usually claim that "in Schaan" is a predicate or paraphrase "She is in Schaan" into "She is in a state of being in Schaan". Both approaches are transparently artificial.

In spite of bearing marks of outstanding ontological importance, locative sentences have been almost never discussed in the literature of ontology.

1.6 The present essay intends to cover this gap. It forms a first, still very preliminary, step into combination ontology.

Namely, combination can be treated as location plus connection. In turn, combination metaphysics can be made by experience with the idea that existence is combination, i.e. location plus connection, plus condensation plus stabilization (plus, perhaps, something else). In short:

$$
\begin{array}{ll}
\text { combination } & =\text { location }+ \text { connection } \\
\text { existence } & =\text { combination }+ \text { condensation }+ \text { stabilization }+\ldots
\end{array}
$$

1.7 The idea which I am going to develop here is as follows: $x$ is located in $y$ if and only if each of its parts is in $y$.

\footnotetext{
${ }^{3}$ Cf. Brückner [2]

${ }^{4}$ Cf. Kahn [5]
} 
1.8 The paper is organized as follows: I start with a general and brief overview of verb-type-ontologies, stressing the importance of the locative one. Next, three main relavant formal theories - of preorders, of mereologies as well as Leśniewski's Ontology — are presented. They are shown to be inadequate to formalise location.

In this survey a special emphasis is put on premereologies intermediate between classical mereologies and preorders. Premereology seems to be very useful in the field of ontology and metaphysics as the first, purely logical, approximation of the idea of condensation, i.e. the internal strength of unifying connections.

Next, I will pass to a discussion of locative ontologies, introducing them as a generalization of preorders, which fill in certain gaps occurring in both mathematical and philosophical approaches to orders. The bulk of locative ontology is presented in the Parts II and III, where locative orders are introduced and related to more familiar structures outlined previously. At the end, the philosophical content of locative ontology is presented and, finally, several cases of location in some important domais are pointed out.

1.9 The present paper is an essay in mathematical philosophy ${ }^{5}$ : its problems are philosophical, its procedure is mathematical. In particular, in the exposition of feel free to behave like in mathematical study.

The work has two aims: a philosophical one - to clarify one of the most important variants of verb-type-ontology, and a mathematical one to enlarge the body of commonly known theories of orders.

\section{VERB-TYPE-ONTOLOGIES}

2.1 The best way, I think, to introduce a variety of verb ontologies is to introduce a bit of grammar. Indeed, verbs, including the verb "to be", play a crucial role in generating verb ontologies.

2.2 Let's start with a list ${ }^{6}$ of the kernel affirmative English sentences.

Hereafter, $\mathrm{N}$ denotes nouns, $\mathrm{A}$ - adjectives, $\mathrm{V}$ - verbs, $\mathrm{P}$ - prepositions, D - phrases of description, qualification or classification, $\mathrm{M}$ -

\footnotetext{
${ }^{5}$ Recall Leibniz-Russell-Łukasiewicz's program of mathematical philosophy.

${ }^{6}$ I have here followed, mutatis mutandis, Z. Harris and C. Kahn's list, cf. Kahn's [5], relying on my [12].
} 
mereological phrases, L - locative phrases, S sentences. Star (or copula) * is reserved to the verb "to be".

\section{V-SENTENCES}

with verbs not reducible to the verb "to be"

They are state or processual sentences of two sorts:

Intransitive

NV John sleeps.

Transitive

NVN John loves Mary.

NVPN John is looking at Mary.

\section{BV-SENTENCES}

specific for English

expressing the presence and continuity of processes

$\mathrm{N} * \mathrm{D}(\mathrm{V}) \quad$ John is sleeping.

$\mathrm{N} * \mathrm{D}(\mathrm{VN}) \quad$ John is loving Mary.

$\mathrm{N} * \mathrm{D}(\mathrm{VPN}) \quad$ John is looking at Mary.

\section{B-SENTENCES}

with "to be" as their verb

They come in so rich a variety that some people believe that "is" in general is not a proper verb but only a formal copula.

\section{NOMINAL}

$$
\text { D-type }
$$

$\mathrm{N} * \mathrm{~A} \quad$ John is old.

$\mathrm{N} * \mathrm{~N} \quad J o h n$ is a man.

$\mathrm{N} * \mathrm{AN} \quad$ John is a good man. 


\author{
M-type \\ $\mathrm{N} * \mathrm{MN} \quad$ A roof is a part of a house.
}

Possessive

$\mathrm{N} * \mathrm{D}(\mathrm{N}) \quad$ This book is mine.

\title{
ADVERBIAL
}

$\mathrm{N} * \mathrm{D}_{1}\left(\mathrm{VD}_{2}\right) \quad$ John is sleeping silently.

\section{PASSIVE}

$\mathrm{N} * \mathrm{D} \quad$ John is loved.

\section{LOCATIVE}

$\mathrm{N} * \mathrm{PN} \quad$ John is at home.

$\mathrm{N} * \mathrm{~L} \quad$ John is here.

$\mathrm{N} * \mathrm{~L}(\mathrm{PN}) \quad$ John is with Mary.

\section{IDENTITY}

$\mathrm{N} * \mathrm{~N}$

The Evening Star is the Morning Star.

$\mathrm{N}(\mathrm{S}) * \mathrm{~N}(\mathrm{~S}) \quad$ My love is my life.

$\mathrm{N} * \mathrm{~S} \quad$ John's fear is: I have cancer.

$\mathrm{S} * \mathrm{~S} \quad$ That John has AIDS is: John has illness more dangerous than cancer.

\section{EXISTENTIAL}

$\mathrm{N} * \quad$ John is. 
2.3 There is a clear correspondence ${ }^{7}$ between the above spectrum of kernel affirmative statements and the main kinds of ontologies.

$\mathrm{B}$ - and $\mathrm{BV}$-sentences correspond in general to change and process ontologies.

For B-sentences the story is much more complex. Both being and the verb "to be" are indeed said and interpreted in various ways. Nominal sentences of D-type correspond to object-property ontology as well as to ontologies of attributes, predicates and multiplicities. M-type sentences generate mereologies, locative statements generate locative and combination ontologies. Adverbial and possessive sentences are connected with ontology of states, whereas existential statements with existential ontology ${ }^{8}$.

Which specific ontology, if any, is connected with identity statements remains unclear.

2.4 In sum, the spectrum of ontologies coincides with the variety of kernel sentences. It seems that each language, when developed sufficiently, enables us to express ontological ideas in a very economical way: different means for different pictures of the world.

2.5 In spite of their differences, the many uses of the verb "to be" all have something in common.

"Is" is not ambiguous. Also, it is not an empty, purely formal, copula.

It is rather a very general verb, open for particularization and variation.

2.6 Its mechanism can be made fairly clear by observing that the kernel sentences are, in fact, generated by the main questions we use to express our curiosity: What? Who? Which? When? Where? How? Why? In which way? etc.

Classifying information we obtain the following variants of the verb "is":

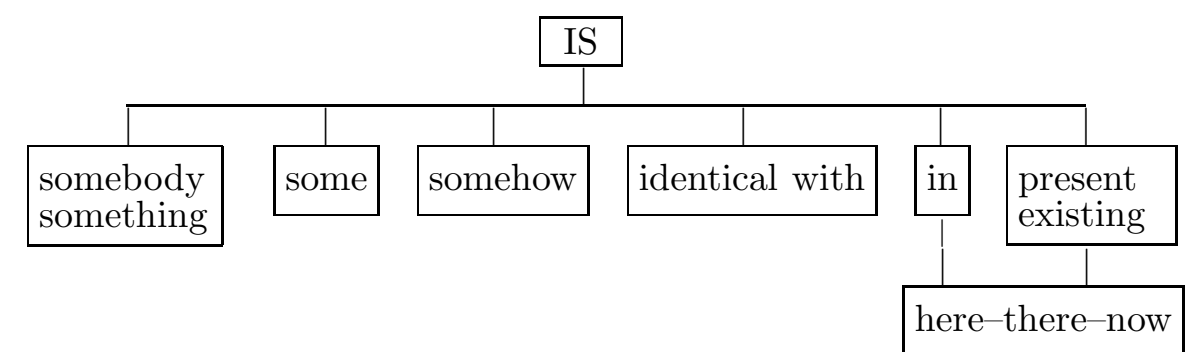

\footnotetext{
${ }^{7}$ For details cf. [13] and [23].

${ }^{8}$ In particular, with so-called existential thomism of Gilson. Cf. Krạpiec [6].
} 
The last two variants clearly generate mereological, collective and locative perspectives on the world.

2.7 It is useful to compare the background of the well established mereological formalisation of the verb "to be" with the locative approach, which we are going to study here.

The common factor of both variants lies in the question-answer pair where - in, which is common to them. On the other hand, their difference can be made clear by connecting the pair who/what - somebody/something (a part of) with the mereological use of "is", whereas the pair where/in which way - present-existing (by or through location) is connected with the locative variant of "is".

Both variants have thereby something in common, but they also differ in a quite sharp way. As it will be made clear later on, locative ontology, although close to mereology, is not reducible to it.

\section{VERB-TYPE-ONTOLOGICS}

3.1 I shall use the term "ontologic" to refer to the formal counterpart of ontology.

Usually, a given ontology generates a bundle of connected ontologics, its fairly complete formal developments.

3.2 Verb ontologics can be divided into two big families: transformation or process ontologics, generated by $\mathrm{V}$-sentences, which formalize change ontologies, and be-logics, generated by B-sentences and formalizing be-ing ontologies.

By the nature of the verb "to be", the most general be-logic is the general theory of relations ${ }^{9}$

3.3 The first step towards formalization is introduction of a suitable notation.

In principle, the symbol $E$ will be used instead of the verb "is" in its most general reading: is related to. I.e., for arbitrary items $x$ and $y$, and for appropriate variant of "is", the expression " $E(x, y)$ " means " $x$ is $y$ ". Following the syntax of natural languages we shall usually write " $x E y$ "

\footnotetext{
${ }^{9}$ Like Schröder and Tarski's theory, cf. [24] and [28]. Notice that in the 1980s the general, set-theoretical theory of relations was revived under the name theory of generalized quantifiers. cf. van Benthem [1] and Westerståhl [29].
} 
instead of " $E(x, y)$ ". Notice, however, that in the most general part of the present paper the symbol $E$ denotes, in fact, an arbitrarily chosen, but fixed binary relation.

3.4 Two types of be-logics should in general be distinguished: standard or elementary, and non-standard or propositional ones. Both assume classical quantificational logic. The difference is syntactical.

In the elementary approach we treat $E$ as a distinguished two-place predicate letter, which builds up atomic formulas only. These formulas, in turn, are used to build up complex formulas by means of the standard classical logical connectives: negation $-\neg$, conjunction $-\wedge$, disjunction $-\vee$, implication $-\rightarrow$, equivalence $-\leftrightarrow$, and minor and major quantifiers: $\exists$ and $\forall$.

Therefore, in the standard case complex formulas are build up only by means of logical connectives. The primitives of a theory, its specific symbols, occur only in terms, if any, and in atomic formulas. For example, the expression $E(x, y)$ is allowed, but $E(E(x, x), z)$ is not. This might be considered an unjust limitation, for the statement "That $x$ is $y$, is $z$ " looks quite reasonable.

On the other hand, in the non-standard, propositional approach by means of $E$ we can build both atomic and complex formulas. There the expression $E(E(x, y), z)$ is well-formed.

3.5 People usually follow the standard approach, in which belogics are simply elementary theories of specific binary relations. By specification we obtain inter alia:

Set-theoretical ontologics; here $E$ equals $\in$ (to be member of), i.e., $x E y:=$ $x \in y$.

Inclusion, or Boolean algebraic ontologics, by considering "to be included in". Here $E$ equals $\subset$, i.e., $x E y:=x \subset y$.

Mereological ontologics, or simply mereologies, by considering "to be a part of". Here $E$ equals $<$, i.e., $x E y:=t x<y$.

Predication ontologics, or predicate calculi by considering "to be predicated by". Here $E$ equals predication, i.e., $x E y:=y(x)$.

Attribution ontologics or property calculi by considering "being an attribute of". Here $E$ equals attribution, i.e., $x E y:=y[x]$.

Nominal identity ontologics, by considering "to be identical with". Here $E$ equals identity, i.e., $x E y:=(x=y)$.

3.6 By comparison of the above list of the main kinds of belogics occurring in the literature with the previous list of the main be-ontologies we 
note several gaps in the first list.

The most urgent is the lack of suitable locative ontologics. In what follows, then, I shall try to fill this gap.

3.7 Finally, let's outline ${ }^{10}$ very briefly the general scheme defining in each case, i.e. for any arbitrarily chosen but fixed relation $E$ its basic ontological notions:

$x$ is $\boldsymbol{E}$-being $:=$ For some $y, x$ is $y: \exists y x E y$. Either more generally

$x$ is $\boldsymbol{E}$-being $:=$ For some $y, x E y$ or conversely for some $y, y E x$ :

$$
(\exists y x E y) \vee(\exists y y E x)
$$

A being is everything which is something or which is of something.

To define the being we need a collecting operator: $\{x: A(x)\}$ denotes the collection of all objects satisfying the condition $A$. Now,

the $\boldsymbol{E}$-being $:=\{x: \exists y x E y\}$ or more generally $\{x:(\exists y x E y) \vee(\exists y y E x)\}$.

The being is the collection of all beings.

To define Being we need a unifying operator: $[x: A(x)]$ denotes the unity of all objects satisfying the condition $A$. Now,

E-Being $:=[x: A(x)]$ or more generally $[x:(\exists y x E y) \vee(\exists y y E x)]$.

Being is the unity of all beings.

3.8 The basic idea is quite natural: beings are items which are, i.e., objects of is - connection.

Observe that in this way we connect verb ontologies with qualitative ones.

3.9 In conclusion, investigating particular be-logics we indeed investigate suitable ontologies.

\footnotetext{
${ }^{10}$ For details cf. [15].
} 


\section{RUDIMENTARY DEFINITIONS}

4.1 Hereafter, I am following the standard, elementary approach. I.e., I am working in a suitable version of the classical predicate calculus with identity $^{11}$, with one non-logical primitive - a binary predicate letter $E$.

In general $E$ should be treated as denoting any arbitrarily chosen but fixed binary relation.

4.2 The standard mathematical notions are used without any ceremony.

In particular the converse of $E, E^{-1}$, is defined by the condition $x E^{-1} y:=$ $y E x$, whereas the superposition of two binary relations $E$ and $E^{\prime}, E \circ E^{\prime}$, is defined by:

$$
x E \circ E^{\prime} y:=\exists z\left(x E z \wedge z E^{\prime} y\right) ;
$$

$x$ is in the $E \circ E^{\prime}$ relation to $y$ if and only if some object $z$ mediates between them: $x E z$ and $z E^{\prime} y$.

4.3 Recall that the following kinds of binary relations are of primary mathematical interest: the transitive, symmetric, antisymmetric and reflexive ones. By their combination we obtain the most important mathematical orders: equivalences, preorders and partial orders.

For readers's convenience I shall repeat below their usual definitions.

$E$ is transitive, if it fulfils the following transitivity condition:

$\mathbf{T}$

$$
\forall x \forall y \forall z(x E y \wedge y E z \rightarrow x E z)
$$

For any $x, y$ and $z$, if $x$ is related to $y$ and $y$ to $z$ then $x$ is related to $z$ as well.

NB. It is a well-established custom, which I will also follow, to omit prefixed general quantifiers.

$E$ is symmetric, if it fulfils the following symmetricity condition:

S

$$
x E y \rightarrow y E x
$$

If $x$ is related to $y$, then also conversely, $y$ is related to $x$.

Usually two types of opposite condition are introduced:

\footnotetext{
${ }^{11}$ Cf. any logical textbook, for example Mendelson [11].
} 
Asymmetry

AS

$$
x E y \wedge y E x \rightarrow x=y
$$

Anti-symmetry

$\mathbf{A}-\mathbf{S}$

$$
x E y \rightarrow \neg(y E x)
$$

$E$ is anti-symmetrical, if it is not reversible.

$E$ is reflexive, if it fulfils the following reflexivity condition

$\mathbf{R}$

$x E x$

Anything is related to itself.

Recalling our ontological interest we can note that for "is" of identity reflexivity becomes the Identity Principle: Everything is self-identical.

4.4 Let me point out also that usually relations are considered only in restriction to a given or presupposed class $U$.

4.5 The smallest reflexive relation on a given set $X$ is named its diagonal and is denoted by $\Delta_{X}$.

$$
\Delta_{X}:=\{\langle x, x\rangle: x \in X\}
$$

4.6 By combination of the above conditions we obtain:

Equivalence relations - these are just the reflexive, transitive and symmetric relations:

\section{EQ}

$$
\mathbf{R} \wedge \mathbf{T} \wedge \mathbf{S}
$$

Preorders - these are just the reflexive and transitive relations:

PO

$$
\mathbf{R} \wedge \mathbf{T}
$$

Partial orders - these are just the transitive and asymmetric relations: POR

\section{$\mathbf{T} \wedge \mathbf{A S}$}

Strict partial orders - these are just the transitive and anti-symmetric relations:

\section{SPOR}

$$
\mathbf{T} \wedge \mathbf{A}-\mathbf{S}
$$


4.7 One general assumption: $E$ is hereafter, if not additionally specified, an arbitrarily chosen binary relation on a fixed universe $U$. Usually, the universe parameter is omitted. For example, the diagonal of a given $U$ is denoted simply by $\Delta$ instead of $\Delta_{U}$.

4.8 Relations are compared by means of inclusion, i.e., $R \leqslant S$ iff the extension of $R$ is included into the extension of $S$.

4.9 Recall the following nice characterization of the above types of relations (cf. Cohn [3]):
(1) $E$ is transitive
iff $E \circ E \leqslant E$, or $E^{2} \leqslant E$
$E$ is symmetric
iff $E \leqslant E^{-1}$, or $E=E^{-1}$
$E$ is asymmetric
iff $E \cap E^{-1} \leqslant \Delta$
$E$ is antisymmetric
iff $E \cap E^{-1}=\emptyset$
$E$ is reflexive
iff $\Delta \leqslant E$
$E$ is an equivalence
iff $\Delta \cup E^{2} \leqslant E=E^{-1}$
$E$ is a preorder
iff $\Delta \cup E^{2} \leqslant E$
$E$ is a partial order
iff $E^{2} \leqslant E$ and $E \cap E^{-1} \leqslant \Delta$
$E$ is a strict partial order
iff $E^{2} \leqslant E$ and $E \cap E^{-1}=\emptyset$

4.10 The above theorem suggests that the most natural kinds of relations are characterizable by suitable conditions of comparison. Indeed, reflexivity of $E$ means that the diagonal relation is weaker than it: $\Delta \leqslant E$; transitivity of $E$ that $E^{2} \leqslant E$, etc.

The most natural relational axioms are therefore conditions of comparison.

4.11 Hereafter the following two conventions are in use:

A For given relations $Q$ and $R$ their suitable comparison conditions are as follows:
$Q R$
$Q \leqslant R$

QR

$Q=R$

B For any relational condition $\phi, \phi \mathbf{S}$ denotes the class of its models:

$$
\phi \mathbf{S}:=\{\langle U, E\rangle:\langle U, E\rangle \text { satisfies } \phi\}
$$


In particular, TS denotes the class of all transitive structures, RS the class of all reflexive structures, POS - the class of all preorders, etc.

4.12 Let me mention also filters and ideals. These are two extremely useful order-concepts.

For a given $x$, by a relation filter $[x)$ generated by $x$ we mean the class of all objects related to $x$, whereas a suitable relation ideal $(x]$ is the family of all objects to which $x$ is related. In symbols:

$$
\begin{aligned}
& {[x):=\{y: x E y\}} \\
& (x]:=\{y: y E x\}
\end{aligned}
$$

Observe that $x$ belongs to both sets iff $E$ is reflexive. Therefore, for reflexive $E$ it is useful to distinguish the body of a filter from the filter itself:

$$
[x]:=\{y: x E y \wedge x \neq y\}
$$

and likewise the body of the ideal from the ideal itself:

$$
(x):=\{y: y E x \wedge x \neq y\} .
$$

To explain our terminology recall Leibnizian idea of the body of a whole as collection of all items subordinated to whole's central element. Expressing this idea in order-terms we see that the body of an ideal $(x]$, i.e. $(x)$, plays indeed the role of $x$-body.

4.13 Finally notice that the most reasonable mathematical candidates for the formalization of the "is in" of locative ontology are preorders or partial orders. You will see, however, that our well-motivated candidate is different, though quite similar.

4.14 In order to introduce locative ontology in a natural way, I will start by collecting certain basic observations concerning well-established and commonly known mathematical and mereological structures.

First I will discuss preorders, next mereologies, passing finally to the proper topic of the paper.

\section{PREORDERS}

5.1 Observe first that the condition

$$
x P y:=\forall z(z E x \rightarrow z E y) \text {, i.e., } x P y:=(x] \subseteq(y]
$$


defines, for any $E$, its conjugate preorder:

(2) $P$ is a preorder, i.e., a reflexive and transitive relation ${ }^{12}$.

Proof. We obtain this immediately, after suitable substitutions, by the identity and transitivity laws for classical implication.

Indeed, $x P y$ iff $\forall z(z E x \rightarrow z E y)$, whereas $x P y \wedge y P u$ iff $\forall z(z E x \rightarrow$ $z E y) \wedge \forall z(z E y \rightarrow z E u)$ iff $\forall z((z E x \rightarrow z E y) \wedge(z E y \rightarrow z E u))$, hence $\forall z(z E x \rightarrow z E u)$, i.e., $x P u$.

5.2 What is the right way of reading $P$ ?

For the ontological understanding of $E$ ( $E$ equals $i s$ ), $x P y$ means that everything which is $x$ is also $y$. This sounds like quite a good expression of the ontological presupposition of the part-whole relation. For this reason, $x P y$ is read: $x$ is a part of $y$.

Notice that in the next chapter stronger mereological part-whole relations will be introduced.

5.3 Let us now assemble a list of all binary relations defined by means of generally closed implications connecting formulas from the list: $z E x, z E y$, $x E z, y E z$ in all possible ways.

There are exactly eight such possibilities. Four of them define converses of the other four. Hence only four definitions are interesting.

The above definition of $P$ is one. The remaining three are the following: $x C y:=\forall z(y E z \rightarrow x E z)$, i.e. [y) $\subseteq[x)$, which is read: $x$ is covered by $y$ $x H y:=\forall z(z E x \rightarrow y E z)$, i.e. $(x] \subseteq[y)$, which is read: $x$ houses $y$ $x D y:=\forall z(y E z \rightarrow z E x)$, i.e. $[y) \subseteq(x]$, which is read: $x$ dominates $y$

As a matter of fact, the four relations indicated above correspond to all cases of inclusion between $x$ and $y$ - filters and ideals: $[x),(x]$ compared with $[y),(y]$.

As regards reading, the above proposals are intended not to be ad hoc. For example, to justify covering think of $E$ as enveloping, i.e., $y E z$ means: $z$ is an envelope of $y$ or $y$ is enveloped by $z$. Now, $y$ covers $x$, if each envelope of $y$ is at the same time envelope of $x$. Isn't it?

5.4 Notice that $C$, like $P$, is in general a preorder:

\footnotetext{
${ }^{12}$ Strictly speaking we should parametrize $P$ by writing $P_{E}$. Remember, however, that by our previous convention $E$ is fixed, hence we can omit it. The proof given in the text is very elemntary. I am giving it here with all details as paradigmatic case to be free to omit such elemntary arguments in the future.
} 
(3) $C$ is a preorder relation.

5.5 As a matter of fact, both $P$ and $C$ are characteristic preorder relations. Namely, by comparison of our primitive relation $E$ with $P$, or with $C$, we can characterize preorders.

To this end observe first

(4) i) $E$ is reflexive iff $P \leqslant E$ iff $C \leqslant E$

ii) $E$ is transitive iff $E \leqslant P$ iff $E \leqslant C$

Proof. I will check only two of the four claimed equivalences, the remaining two leaving to you.

Ad i) If $E$ is reflexive, i.e., $x E x$ then because $x P y$ implies $x E x \rightarrow x E y$, we obtain that $x E y$. Hence $P \leqslant E$.

Conversely, if $\forall x \forall y(x P y \rightarrow x E y)$, then $x P x \rightarrow x E x$. But, by (2), $P$ is reflexive. Hence $x E x$, i.e., $E$ is reflexive as well.

Ad ii) Let $E \leqslant C$, i.e., $\forall x \forall y(x E y \rightarrow x C y)$. Hence $\forall x \forall y(x E y \rightarrow$ $\forall z(y E z \rightarrow x E z))$. This, by classical logic, is equivalent to $\forall x \forall y \forall z(x E y \rightarrow$ $(y E z \rightarrow x E z))$, which is the transitivity condition for $E$.

The reverse implication can be checked simply by reversing the above reasoning.

As immediate corollary we obtain the following characterization of preorders

(5) $E$ is a preorder relation iff $E=P$ iff $E=C$ iff $P=E=C$.

5.6 There is an important philosophical problem to find the right axiomatization of the part-whole relation.

The problem clearly has at least two components: one purely formal concerning the relation to be a part of, and the other, more essential, though still formal - concerning wholeness.

Therefore, if we take preorders to be the first and very general approximation of the relation to be a part of, we can claim that the formula ${ }^{13}$

$$
P=E
$$

saying: to be is to be a part of, by its generality, formalizes (onto)logical mereology. In such a case, (onto)logical mereologies are simply preorders.

\footnotetext{
${ }^{13}$ Similarly for equivalent axioms: (EC) $E=C$, and (PEC) $P=E=C$.
} 
5.7 Equivalence relations are preorders of strong form. Notice that they can be characterized in a way similar to one given in (5).

First recall that by (1), $E$ is symmetric iff $E=E^{-1}$. Combining this with (5) we obtain the following characterization of equivalence relations:

(6) $E$ is an equivalence relation iff $E^{-1}=P$ iff $E^{-1}=C$ iff $P=E^{-1}=C$.

Proof. First notice that $E$ is reflexive, or transitive, or symmetric, or an equivalence relation iff its converse $E^{-1}$ fulfils the respective condition.

I will check only the first equivalence in our claim, for the remaining two are similar.

Let $E$ be a relation of equivalence. By its symmetricity $E=E^{-1}$, whereas by its being preorder $E=P$. Hence $E^{-1}=P$.

Conversely, let $E^{-1}=P$. By (5), $E^{-1}$ is a preorder relation, hence by the first observation of the proof, $E$ is a preorder as well. Repeating (5) we obtain $E=P$. Therefore $E=E^{-1}$, hence $E$ is symmetric. In conclusion, $E$ is a symmetric preorder, i.e., an equivalence relation.

5.8 To conclude, relations $P$ and $C$ are indeed characteristic preorders.

As regards the remaining two relations $H$ and $D$, they are, in a sense, connected with symmetricity.

First of all, observe that they connect symmetricity with reflexivity:

(7) i) $E$ is symmetric iff $H$ is reflexive iff $D$ is reflexive

ii) $E$ is symmetric iff $P=H$ iff $C=D$

Proof. The case (i) and the right-hand implication in (ii) follow immediately from the definitions.

For the left-hand implication in (ii), assume that $P=H$. Hence $H$ is a preorder, for $P$ is. Therefore $H$ is reflexive. By (i), $E$ is symmetric, as required.

Next observe

(8) If $E$ is symmetric, i.e., if $H$ and $D$ are reflexive, then both $H$ and $D$ are transitive, hence preorders.

5.9 Combining the above results with the previous ones we finally reach

(9) $E$ is an equivalence relation iff $E=P=C=H=D$.

Therefore, in the case of equivalence relations all 10 relations involved: $E, P, C, H, D$ and their converse relations, coincide. 
Equivalence relations are very regular indeed!

5.10 Query. It is easy to see that four-element equality: $P=C=H=D$ doesn't entail that $E$ is equivalence.

It characterizes thereby some broader class of symmetric relations. Which one?

5.11 Using the four relations: $P, C, H$ and $D$, and in particular the first two, we can obtain quite aesthetic characterizations of the standard mathematical order-relations.

Notice, however, a remarkable gap. Until now we didn't compare $P$ and $C$ in an immediate way, but only by means of the basic relation $E$. It will become clear shortly that such immediate comparison means location.

5.12 Final remarks concerning partial orders. First of all, partial orders and preorders overlap. As a matter of fact we should distinguish reflexive partial orders, i.e. asymmetric preorders, and irreflexive ones, i.e. partial orders which are not preorders.

Interrelations between relevant classes of relations can be presented as follows:

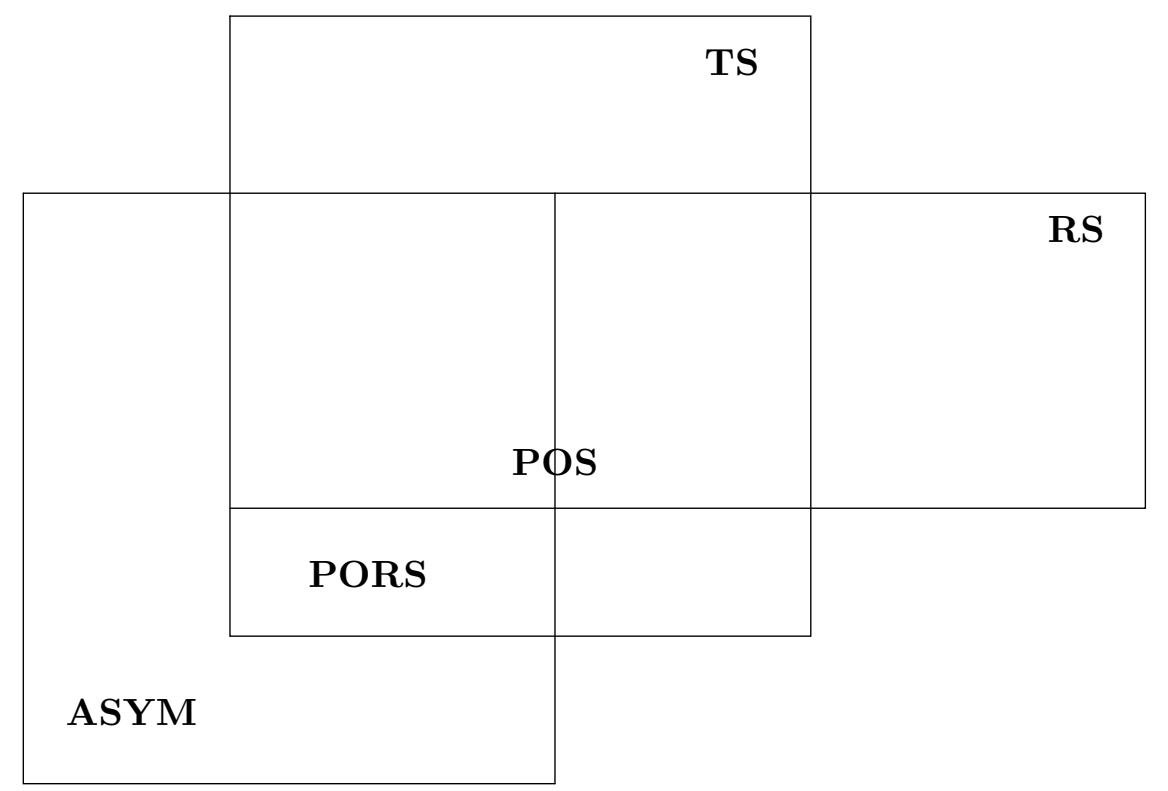

Fig. 1. 
5.13 The basic fact about partial orders of the first type, i.e. asymmetric preorders, is that they are extensional, i.e., fulfil the extensionality condition:

Ext

$$
x=y \leftrightarrow \forall z(z E x \leftrightarrow z E y)
$$

In fact, we can prove even more:

(10) If $E$ is reflexive and asymmetric, then $E$ is extensional.

Proof. The right-hand implication is due to the extensionality of classical logic.

Conversely, assuming the right-hand equivalence $\forall z(z E x \leftrightarrow z E y)$, by suitable particularization and using logic, we obtain $x E x \wedge y E y \leftrightarrow x E y \wedge$ $y E x$. Hence, by reflexivity of $E, x E y \wedge y E x$. Applying now the asymmetry condition AS we reach the conclusion: $x=y$.

\section{PREMEREOLOGIES}

6.1 Proper mereologies consider both sides involved: the relation to be a part of and wholes, trying to approach the latter by means of the former.

6.2 We start by introducing a few additional notions.

The first two should be understand as general, i.e. as not specifically mereological.

$$
x O y:=\exists z(z E x \wedge z E y), \quad \text { i.e. }(x] \cap(y] \neq \emptyset
$$

$x$ overlaps with $y$ iff some object $z$ is both $x$ and $y^{14}$.

$$
x D S y:=\neg(x O y)
$$

$x$ is discrete from $y$, if they two do not overlap.

Now we are ready to define the mereological order relation $M-i s$ a (mereological) part of - by the condition saying that everything overlapping a part overlaps also a whole:

$$
x M y:=\forall z(z O x \rightarrow z O y), \quad \text { i.e. }[(x]) \subseteq[(y])
$$

\footnotetext{
${ }^{14}$ Recall that I am consequently using the ontological reading of $E$ by "is".
} 
By definition

$$
x M y \text { iff } \forall z(z D S y \rightarrow z D S x)
$$

For brevity's sake, the relation $M$ will often be read as "meeting relation". I.e., $x M y$ is read: $x$ meets $y$.

6.3 Previously, for any given binary relation $E$ its conjugate part-relation was defined. Is $M$ definable after this manner?

Yes. For a given relation $E$ its meeting relation $M$ is equal to the part-relation $P$ taken, however, for overlapping $O$ instead of the primitive relation $E$. I.e., using parameters we can write: $M_{E}=P_{O}$, or more exactly: $M_{E}=P_{O_{E}}$.

A question arises: Does $M=P$ hold in general?

Later on, cf. (15), you will see that the answer is: No, it isn't.

\section{Mereological Condensation}

6.4 The crucial mereological axiom, common for standard ${ }^{15}$ mereological systems including suitable calculi of individuals, is the following axiom of mereological condensation:

ME $E=M$, i.e., $\forall x \forall y(x E y \leftrightarrow x M y)$

To be means to stand to something in the mereological meeting relation.

The axiom ME defines the class of premereologies, a realm intermediate between proper mereologies ${ }^{16}$ and preorders.

6.5 Similarly to $P$ and $C$, and for similar reasons

$M$ is a preorder.

Hence, if $E$ is premereological, i.e. if it satisfies $\mathbf{M E}$, then $E$ is a preorder relation as well. Therefore

All premereologies are preorders.

We may ask now, which preorders are premereological?

\footnotetext{
${ }^{15}$ For references a reader is addressed to P. Simons' treatise [25] or to M. Libardi's survey [10].

16 I.e. classical extensional mereology, some of its subsystems and Leśniewski's Mereology [7], [8] and [9]. Cf. Simons [25], Libardi [10].
} 
It is easy to check that not any preorder is such. We will see that in fact premereologies show some similarity to Boolean structures, whereas proper mereologies simply imitate them.

6.6 By (5) and (12)

(14) If $E$ is premereological, i.e. if it satisfies $\mathbf{M E}$, then $M=E=P$

Therefore, whereas $E=P$ is the characteristic equation for preorders, $M=P$ seems to be characteristic for premereologies. In general it is not true. Consider, for example, the empty relation. But for preorders it is.

(15) If $E$ is a preorder, then $E$ is premereological iff $M=P$.

Proof. The right-hand implication is implicit in (14).

For the reverse implication, notice that $E=P$, for $E$ is a preorder. By the second assumption, $M=P$, hence $E=M$, i.e., $E$ is premereological, as required.

In conclusion, in the realm of preorders premereologies identify the (onto)logical part-relation with meeting. In this case, only parts meet wholes.

\section{Overlapping}

6.7 We are going now to collect a few elementary properties of overlapping, in particular those which compare it with the other relations under investigation.

From the definitions we immediately obtain

(16) $O$ is symmetric in general, and reflexive provided $E$ is such.

As regards comparison:

(17) If $E$ is reflexive then $M \leqslant O$ and $P \leqslant O$. Also, as was stated in (4), reflexivity of $E$ implies $P \leqslant E$

(18) If $E$ transitive, then $E \leqslant M$ and, cf.(4), $E \leqslant P$.

Hence

(19) If $E$ is a preorder relation, then $P=E \leqslant M \leqslant O$.

Therefore, taking into account (15), we see that in the realm of preorders premereologies can be characterized by the inequality $(\boldsymbol{M P}) M \leqslant P$. 
6.8 Let us examine the three inequalities from (19): $P \leqslant M, M \leqslant O$ and $P \leqslant O$.

For brevity's sake we limit consideration to the case of preorders, i.e., we assume that $E=P$.

First, observe that in general none of the above inequalities is reversible.

As regards $M \leqslant P$ we know that in the case of preorders it axiomatizes premereologies.

Next, note that in general

$$
O \leqslant M \text { iff } O \text { is transitive. }
$$

Hence, in the case of preorders

$$
O=M \text { iff } O \text { is transitive. }
$$

Notice, that the restriction to preorders is essential, for even in the case of premereologies $O$ has not to be transitive.

6.9 Finally, let's turn to the strongest equivalence: $O \leqslant P$. I.e. $O \leqslant E$, for we work with preorders only.

In this case, by (19), $E=P=M=O$. It is easy to foresee that this collapse of all relations involved onto one relation means that it is an equivalence relation. Indeed

(22) Let $E$ be a preorder. $O \leqslant E$ iff $E$ is an equivalence relation.

Proof. The left-hand implication is well-known. It is, in fact, one of the key cases in the abstraction principle connecting equivalence relations with partitions of sets.

For the reverse implication assume that $E$ is a preorder, and that $x O y \rightarrow$ $x E y$, i.e., $\exists z(z E x \wedge z E y) \rightarrow x E y$. By classical logic, $\forall z(z E x \wedge z E y \rightarrow x E y)$. Putting $z:=y$ we obtain: $y E x \rightarrow x E y$. Hence $E$ is a symmetric preorder. I.e., it is an equivalence relation, as required.

6.10 To sum up: Joining (9) with the previous observation we see that in the case of equivalence relations all relations yet considered collapse: $E=$ $P=C=H=D=M=O$.

Equivalence is indeed regular. Regularity means simplicity. But too much regularity means too much simplification, killing differences as the dessert kills forests.

6.11 Notice that in general, i.e. for any relation $E$, the following laws of monotonicity for overlapping hold: 
(23) $z O x \wedge x P y \rightarrow z O y$ and $z O x \wedge x M y \rightarrow z O y$;

whereas

(24) $z O x \wedge x E y \rightarrow z O y$, provided $E$ is transitive.

If a smaller item overlaps with some third item then the bigger item overlaps with the latter as well.

\section{Condensation Revisited}

6.12 Let us return to the discussion of the mereological axiom ME.

First two definitions - of proper being (related) and being a proper part of:

$$
x P E y:=x E y \wedge x \neq y
$$

$x$ is properly (related to) $y$ iff $x$ is (related to) $y$, but the two differ from each other;

$$
x P P y:=x P y \wedge x \neq y
$$

$x$ is a proper part of $y$ iff $x$ is a part of $y$, but different from it.

In the realm of preorders both notions clearly coincide.

Also, assuming $\mathbf{A S}$ we have the following: if $x$ is properly $y$ then $y$ is not $x, x P E y \rightarrow(y E x)$.

Similarly, in the case of asymmetric preorders, if $x$ is a proper part of $y$ then $y$ is neither $x$ nor a part of $x$ :

$$
x P P y \rightarrow \neg(y E x) \wedge \neg(y P x) .
$$

6.13 Now, is the time to introduce the basic ontological opposition between atoms (simples or elements) and complexes.

For a given relation $E$, an object $x$ is said to be $E$-simpler than $y$, if $x E y$. Next, $x$ is said to be an atom of the relational space $\langle U, E\rangle$, what we write $A(x)$, if no different object is simpler than it, or, using terms from 4.12 , it is without body:

$$
A(x):=\neg \exists z(z P E x), \quad \text { or } \quad(x)=\emptyset .
$$

Out of preorders atoms should carefully be distinguished from simples: $x$ is a simple, if no object is simpler than it, or if it is an atom not simpler than itself.

$x$ is a complex iff it is not an atom, or it has a body:

$$
C(x):=\exists z(z P E x), \quad \text { or } \quad(x) \neq \emptyset .
$$


Atoms, simples and complexes are, for sure, basic notions of any combination, combinatorial or constructional ontology. In spite, or perhaps because of, their obviousness they are rich and fruitful notions, which clearly deserve a very detailed study.

For such an account an interested reader is referred to [19] and [23]. In the next two sections I will employ both notions to characterize premereologies. Later on I will use them to classify locative ontologies.

6.14 We are working, as usual, with preorders. I.e., $E$ is taken to be a preorder relation.

Observe first

If $(x)=\emptyset$ then for any $y, x M y$ implies $x E y$.

Proof. As a matter of fact we will prove this using only the reflexivity of $E$.

Suppose that $x M y$, i.e., (i) $\forall z(z O x \rightarrow z O y)$. But $x$ is an atom, therefore (ii) $\forall z(z O x \leftrightarrow x E z)$. By reflexivity of $E, x E x$. Hence $x O x$. Therefore, by (ii), $x O y$, i.e. $y O x$. Applying again (ii) we obtain $x E y$, as required.

Notice that in the above claim the assumption of the reflexivity of $E$ is essential. Observe first that a simple $x$ meets everything: if $x$ is simple, then for any $y, x M y$. Therefore, (25) is falsified by any frame with simples which are not related to everything.

Combinig (25) with (18) we obtain

(26) If $x$ is an atom, then for any $y: x E y \leftrightarrow x M y$.

In other words, at the very bottom of a preorder, i.e. for its atoms, the axiom ME holds.

Therefore the real difference between preorders and premereologies occurs only in the realm of complexes.

6.15 Hence, let's turn to the realm of complexes.

First a trivial but useful observation:

(27) For a transitive $E$, if $x E y$ then $(x) \subseteq(y)$ and if, in addition, $x$ is complex then also $y$ is complex.

The next claim is more essential

If $x$ is complex then $(x) \subseteq(y) \rightarrow x M y$. 
Proof. Assume that $(x) \neq \emptyset,(x) \subseteq(y)$ and that for arbitrary but fixed $z, z O x$. Hence for some $u, u E z$ and $u E x$.

If $u=x$, then $x E z$. Take $\underline{u} \in(x)$, i.e., $\underline{u} \neq x$ and $\underline{u} E x$. By transitivity of $E, \underline{u} E z$. On the other hand, $\underline{u} \in(x) \subseteq(y)$, i.e. $\underline{u} E y$. Therefore $z O y$. In conclusion, $x M y$, as required.

If $u \neq x$ we repeat the above argument with $u$ instead of $\underline{u}$, reaching the same conclusion.

The above implication in the field of all preorders is not reversible. It can, however, be reversed for premereologies because for them it is equivalent to: $(x) \subseteq(y) \rightarrow x E y$ which, by (27), is reversible for any transitive frame.

Thus we obtain

(29) Let $E$ be premereological and $x$ be complex. Then $x E y \leftrightarrow(x) \subseteq(y)$.

\section{Augmentation}

6.16 The last equivalence fully deserves to be isolated as the principle of augmentation:

AP If $x$ is complex, then $x E y$ iff $(x) \subseteq(y)$.

Complexes grow with bodies!

6.17 It is noticeable that for asymmetric preorders the augmentation principle AP implies the following natural principle of difference:

DP If $x$ is complex, then $x \neq y$ iff $(x) \neq(y)$.

Different complexes differ as to their bodies!

6.18 In the previous section we, in fact, proved:

(30) For each premereology, the augmentation principle AP holds.

Is it equivalent or weaker than premereology axiom, i.e., is $\mathbf{A P}$ equivalent to ME?

6.19 The answer both in general and in the case of preorders is: No, they are not equivalent.

As a matter of fact $\mathbf{A P}$ is weaker than ME. To see this consider two infinitely decreasing, reflexive and transitive chains: 
$z E \ldots x_{2} E x_{1} E x, z E \ldots y_{2} E y_{1} E y$ with common point at infinity related to any point:

$x$

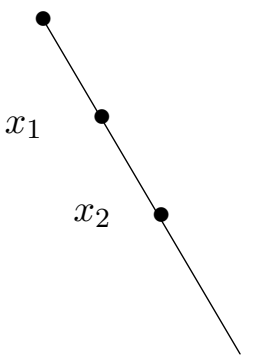

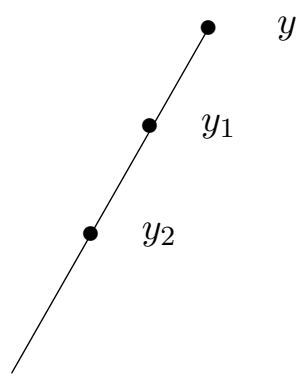

Fig. 2 .

Clearly $x M y$, but $\neg(x E y)$. Hence ME doesn't hold. On the other hand, it is obvious that in our model $\mathbf{A P}$ is valid.

6.20 Note that each augmented structure must be a preorder when restricted to its complexes, but taken together with atoms (if any) it need be neither reflexive nor transitive. In general, the fields of augmented structures and preorders cross each other.

6.21 However, in a rather distinguished case augmentation is equivalent to the premereological characterization of "is". Namely, AP is equivalent to ME in inductive preorders.

A preorder is said to be inductive iff it fulfils the Downard Chain Condition:

DDC Any chain of decreasing items is finite.

In inductive preorders we can define by standard induction the height (or rank) for each object. 
Observe that

(31) Inductive preorders must contain atoms, and that any finite preorder is inductive.

Now we are ready to establish the following theorem:

(32) Let $\langle U, E\rangle$ be an inductive preorder. Then $\langle U, E\rangle$ satisfies $\mathbf{M E}$, i.e., it is a premereology iff $\langle U, E\rangle$ satisfies $\mathbf{A P}$, i.e., it is an augmented structure.

Proof. The right hand implication follows by specification of (29).

For the reverse implication assume that $\langle U, E\rangle$ is an inductive preorder satisfying AP, but not ME.

Hence, for some $x$ and $y$ : $x M y$ but $\neg(x E y)$. By (26) $x$ is complex. Hence, by $\mathbf{A P},(x) \nsubseteq(y)$. Thus, for some $z: z \neq x, z E x$ and $\neg(z E y)$.

We claim that $z M y$. To see this, let's take $u$ such that $u O z$. By (24), $u O x$, for $z E x$. Hence $u O y$, for we supposed that $x M y$. Therefore, for any $u$, $u O z \rightarrow u O y$, i.e., $z M y$.

Thus we fond a such $z$ that: $z P E x, z M y$ and $\neg(z E y)$.

Clearly we can repeat the above procedure again and again, obtaining an infinite decreasing chain: $\ldots z_{i+1} E z_{i} \ldots z E x$, which contradicts the inductiveness of $\langle U, E\rangle$.

6.22 Two corollaries follow:

(33) For any finite preorder, ME is equivalent to AP. In other words, finite preorders are augmented iff they are premereological.

This holds in virtue of (31) and (32).

For the next corollary we need to recall: $A$ (reflexive) tree is any preorder such that each of its elements has at most one immediate predecessor which is different from it.

Immediately from (32) we have

(34) The converse of an inductive tree is premereological iff it is a linear preorder. 


\section{The Scope of ME}

6.23 Now, it is easy to see the power of the condensation axiom ME. Inter alia, it excludes all structures of the form
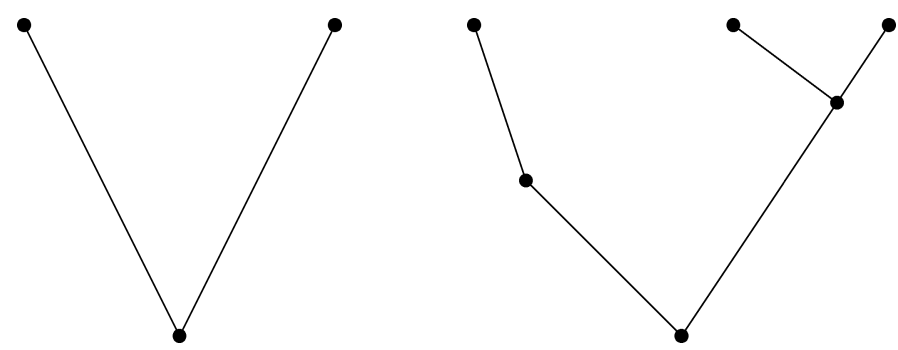

etc.

Fig. 3 .

i.e. converses of all inductive trees different from chains.

For in premereology different complexes differ in their proper parts, which in proper mereology is strengthened to so-called supplementation principles, cf. 6.33.

6.24 The axiom ME also excludes all atomic structures having the smallest element called zero, which are different from chains:

(35) If $\langle U, E\rangle$ is an atomistic, inductive premereology with a zero element then it must be a chain.

For in atomistic premereologies with zero all atoms, i.e. immediate successors of zero, collapse. Next, apply induction.

In the next section we will see that, in fact, zero trivializes premereologies.

Therefore the following structures are also excluded:
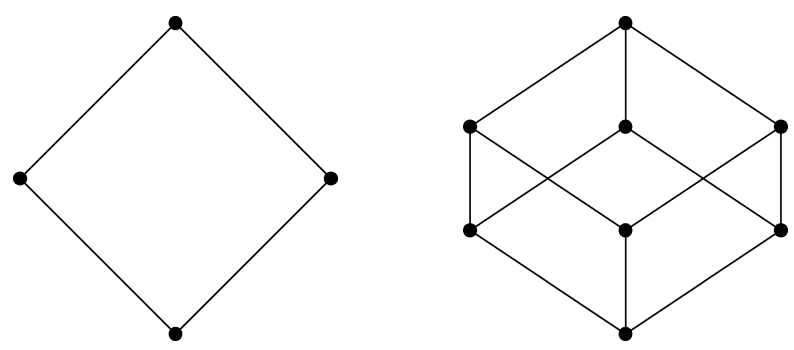

... Atomistic

Boolean

Algebras 

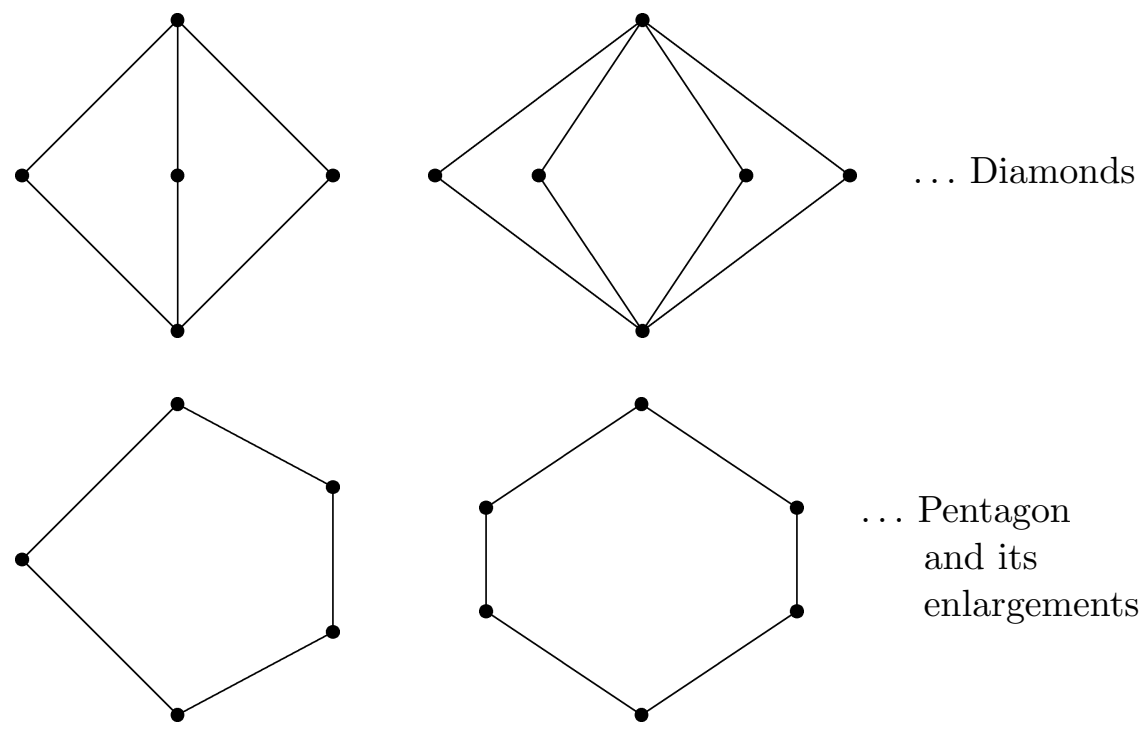

Fig. 4.

Notice that by cancelling zero in the above examples we obtain premereologies.

6.25 As a matter of fact, zero is forbidden in proper premereologies, for it trivializes them.

Indeed, if $z$ is a zero, i.e., if $\forall x z E x$, then $M$ is full: $\forall x \forall y x M y$. Hence, by $\mathbf{M E}$, the starting relation $E$ is also full.

If, in addition, we assume asymmetry condition AS then

(36) Each asymmetric premereological structure $\langle U, E\rangle$ with zero is trivial, i.e., $U$ is a singleton.

\subsection{To sum up:}

$1^{\circ}$ Think that the ordered space $\langle U, E\rangle$ is condensely ordered ${ }^{17}$ by $E$, if its points are connected by $E$ only if their neighbours are also connected by $E$. Now, it is easy to see that that the condensation axiom ME realy does condense structures. In quite a lot of cases it condenses them to chains, or even to full relation structures. In noninductive cases it condenses them to fairly symmetric structures in which different objects can be differentiated from the bottom.

\footnotetext{
17 Condense orderings discussed here should be distinguished from dense orders, i.e. relations enjoying the property that if $x E y$ the for some $z, z \neq x$ and $z \neq y$ and $x E z E y$.
} 
$2^{\circ}$ Preorders seem to catch quite well the formal properties of the relation "to be a part of". Premereologies, in addition, try to characterize wholes as objects having regular internal structures, which is expressed by the principle that complexes grow with their bodies.

This idea is clarified by means of the further mereological axioms.

\section{Infinity}

6.27 Let's return to the proof of the theorem (32).

This makes clear that for preorders the negation of the implication $\mathbf{A P} \rightarrow \mathbf{M E}$, i.e. the conjunction $\mathbf{A P} \wedge \neg \mathbf{M E}$, expresses infinity in a way similar to the well-known formula of Schütte:

$$
\forall x \exists y(x E y) \wedge \forall x \forall y \forall z(x E y \wedge y E z \rightarrow x E z) \rightarrow \exists x(x E x)
$$

Schütte's formula is known to be false only in infinite domains, like the implication $\mathbf{A P} \rightarrow \mathbf{M E}$ we just have investigated.

To be more exact

$$
\begin{aligned}
& \text { Let }\langle U, E\rangle \text { be a preorder falsifying } \mathbf{A P} \rightarrow \mathbf{M E} \text {, i.e. verifying } \\
& \mathbf{A P} \wedge \neg \mathbf{M E} \text {. Then }\langle U, E\rangle \text { is infinite. }
\end{aligned}
$$

6.28 The question "Does augmentation imply condensation?" is indeed very natural. However, to answer it in the negative we must refer to infinity.

Therefore, infinity seems to be deeply involved in the mereological approach to wholes.

\section{Towards mereology}

6.29 As it has been noticed previously, typical further mereological axioms clarify further the mereological structure of wholes.

In this sub-chapter I am going to overview the most standard axioms of this sort. I am, however, not going into details which are easy to find in Simons' and Libardi's books mentioned previously.

6.30 To guarantee ease of definitions people usually introduce the following axiom-scheme of (mereological) comprehension:

CA For each formula $A(x)$, with a free variable $x$ :

$$
\exists x A(x) \rightarrow \exists y \forall z(z O y \leftrightarrow \exists u(A(u) \wedge z O u)) .
$$


If $A$ is satisfiable, then there exists y such that for any $z, z$ overlaps with $y$ iff it overlaps with some object satisfying $A$.

Clearly, assuming AS, $y$ is the union (or fusion) of all objects satisfying $A$ which overlap with $z$.

CA allows the introduction of all usual operations, including intersection, union, complementation, etc.

6.31 Observe that applying it to a tautologous formula we obtain (38) $\exists y \forall z z O y$.

Hence, by AS, we deduce the existence of the universal object $\mathbf{1}$ such that $\forall z z O 1$. Therefore, by definition of $M, \forall z z M 1$. Finally, applying ME, we obtain

(39) $\forall x x E 1$.

We can also prove that

(40) For any family of objects there exists its fusion.

6.32 However, from the ontological point of view. both the existence of 1 as well as the last claim are very suspected.

Ontology deals with all possibilities. But quite a lot of collections seems to be mutually incompatible in such a way that they cannot coexist. Hence they have no fusion, which contradicts (40).

Therefore, I think, mereology with full comprehension deals with a rather special case in ontology. In its full power it, perhaps, is more useful in metaphysics (i.e. ontology of the world) or rather in the quasi-geometrical description of the world.

6.33 For the needs of ontology mereology has thereby to be weakened.

How far? Are premereologies better? Perhaps we should weaken them further?

6.34 Return to mereology. Another group of axioms is introduced to explain, step by step, the characterisation of difference provided by the Augmentation Principle. It includes, among other axioms, several supplementation principles:

The Weak Supplementation Principle:

WSP

$$
x P P y \rightarrow \exists z(z P P y \wedge z D S x)
$$


If $x$ is a proper part of $y$ then some proper part of $y$ is disjoint from it.

The Strong Supplementation Principle:

SSP

$$
\neg(x P y) \rightarrow \exists z(z P x \wedge z D S y)
$$

If $x$ is not a part of $y$ then some part of $x$ is disjoint from $y$.

In the field of sets ordered by inclusion this supplementation is simply the set-theoretical difference $x-y$. This analogy is expressed in

The Remainder Principle:

RP

$$
\neg(x P y) \rightarrow \exists z \forall w(w P z \leftrightarrow w P x \wedge w D S y)
$$

If an object is not part of a second object, then there exists a unique remainder (difference) of the first minus the second ${ }^{18}$.

6.35 All these axioms are introduced in order to increase the similarity of mereologies to Boolean structures.

Fine. But should our ald acquaintance with non-boolean situations be forgotten in metaphysics?

\section{Summary}

6.36 In the present overview of mereology two new classes of structures has been introduced and investigated: premereologies and augmented structures. They both extend mereology, in two slightly different ways, however.

6.37 The relations between types of structures investigated in the present chapter are as follows:

Each mereology is a premereology, which, in turn, is at once a preorder and an augmented structure. Classes of preorders and augmented structures cross each other.

Using self-explaining symbols: MS - for mereologies, PS - for premereologies, POS - for preorders and AS - for augmented structures, these connections can be drawn as follows:

${ }^{18}$ Cf. Simons [25] 


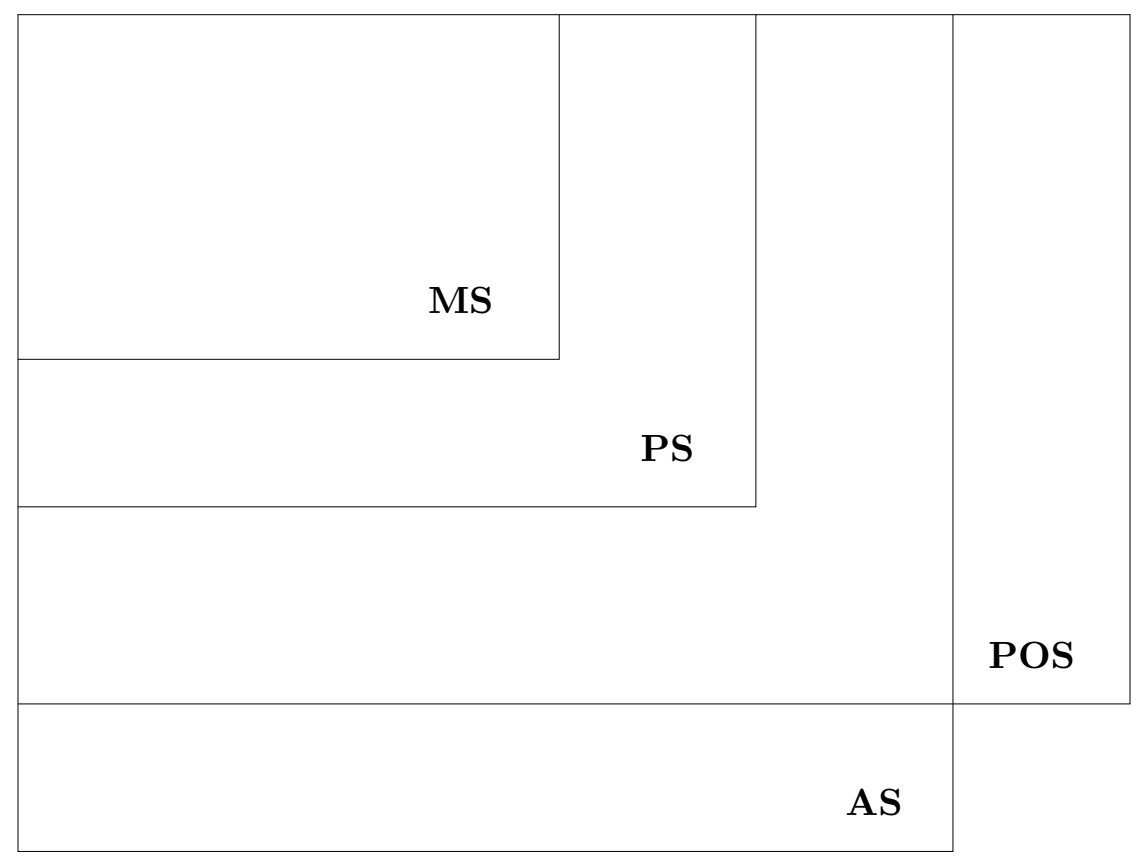

Fig. 5 .

\section{LEŚNIEWSKI'S ONTOLOGY}

7.1 No survey of available theories of ontologically relevant orders can omit Leśniewski's Ontology ${ }^{19}$. It should interest us particularly because Leśniewski constructed it with a strong ontological idea in mind - to grasp the most general and primitive, the deep sense of the verb "is".

Whether, and to what extent, he was successful is a problem for discussion. In particular, it is not clear in which way the formal machinery of Leśniewski's Ontology expresses and is connected with his never published nominalistic philosophy which is known from tradition.

These questions call for attention. They need subtle and extended discussion. Because of shortage of space, here I can only announce it. An interested reader is referred to an accompanying paper "The Nominalism of Leśniewski's Ontology" [18 ], cf. also Smith [26].

\footnotetext{
${ }^{19}$ Cf. Leśniewski [8] and [9]. Cf. also Srzednicki i Rickey [27].
} 
In this chapter, I am only discussing Leśniewski's axiom in relation to the systems introduced above.

7.2 Leśniewski's axiom is as follows:

LON $\quad x E y \leftrightarrow(\exists z z E x) \wedge \forall z \forall u(u E x \wedge z E x \rightarrow u E z) \wedge \forall z(z E x \rightarrow z E y)$

This is, to be sure, a rather long and somehow obscure formula. Usually, the axiom is treated as an implicit definition of singular inclusion. The three conditions on its right-hand side correspond, in a sense, to Russell's condition for definite descriptions: there is at least one $x$, there is at most one $x$, and wherever is $x$ is $y^{20}$.

This rather obvious association can, however, be misleading. Russell's conditions are expressed for a fixed predicate (or formula) which occurs in each of the three conditions involved. This, however, does not hold in our case. On the other hand, there is no straight path from Leśniewski's basic expression " $x E y$ " to the atomic formula of classical logic " $y(x)$ ".

Leśniewski's axiom should thereby be understood not through its supposed genesis, but through logical analysis.

7.3 First of all, the form of the axiom is similar to the one pointed out in the case of preorders and premereologies. Their axioms, we remember, are equations indicating suitable equivalents to the primitive relation $E$, respectively $E=P$ and $E=M$.

Which equation is appropriate for the case of LON?

Observe that all three formulas on the right-side of LON are old friends. The third one is our definition of the part-relation. Therefore, Leśniewski's axiom is of the form $E=P \cap(S \times U)$, where $S$ is a singularity condition:

$$
\begin{array}{lll}
S(x) & :=N(x) \wedge \operatorname{Sol}(x), \quad \text { where } & \\
N(x):=\exists z z E x & & \text { Nonemptiness Condition, and } \\
\operatorname{Sol}(x):=\forall u \forall z(u E x \wedge z E x \rightarrow u E z) & \text { Uniqueness Condition }{ }^{21}
\end{array}
$$

7.4 Observe that in Leśniewski's ontologies

$$
x E y \rightarrow x P y
$$

Hence, by (4), $E$ is transitive. In general, however, it is not reflexive. Hence Leśniewskian ontologies en gros are not preorders. As a matter of

${ }^{20}$ Cf. Simons [25].

${ }^{21}$ In the theory of relations this is known as the anti-Euclidean condition. 
fact, the realm of preorders and the realm of Leśniewskian ontologies cross each other. They cross also with the realm of augmented structures.

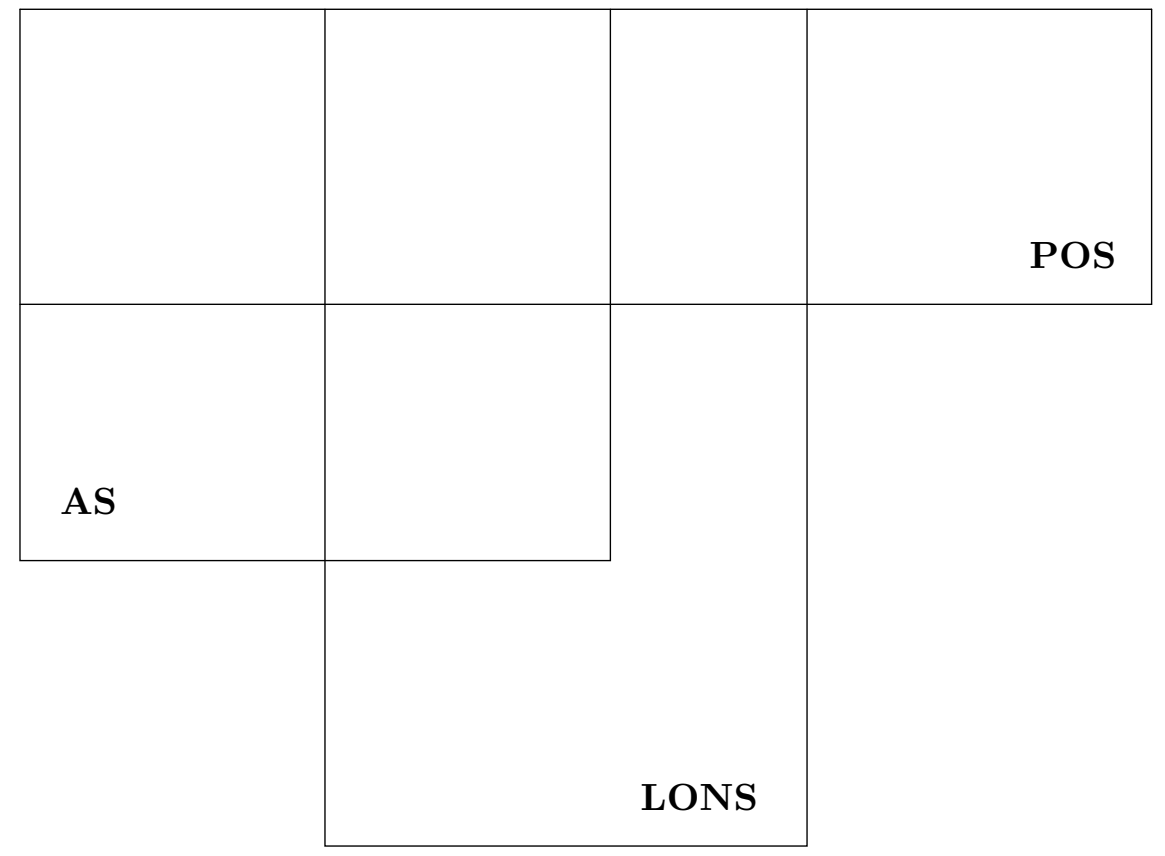

Fig. 6.

7.5 What condition, then, together with transitivity, characterizes Leśniewski's Ontology?

We can find several conditions, including

(42) LON is equivalent to: $\mathbf{T} \wedge(S(x) \leftrightarrow \operatorname{Id}(x)) \wedge(x E y \rightarrow \operatorname{Id}(x))$, where $\operatorname{Id}(x):=x E x$

For the proof, explication and further discussion of Leśniewski's Ontology the reader is referred to [18]. 


\section{PART II. LOCATION}

The most subtle points of formalisation are: first, fix intuition and, next, find adequate and as simple as possible formal means to express and develop it.

Our investigation of location starts with its informal discussion. After fixing intuition several clues concerning location are axiomatized in the relational framework and, next, developed in a very preliminary way.

\section{BASIC CONCEPTS, KINDS AND PROPERTIES OF LOCATION}

\section{The Idea}

8.1 The idea of location is both general and fundamental.

Location has several sides: topological - find a place, take it and fill or cover it; geometrical or physical - locate by fixing (space-time) coordinates, practical - locate successfully and economically, and so on.

Therefore, location has a wealth of connections to such items as measure, similarity, etc.

8.2 Location has also a quite remarkable ontological dimension.

It has been pointed out in chapter 2 that some basic, primitive be-statements are locative ones.

Consider, for example, a sentence: I am here, or its specification: I am in Schaan. This locates me in a nice city of Liechtenstein. But what does this exactly mean?

Clearly, I am a compound item. Hence, if I am in Schaan, then everything of which I am compounded must also be in Schaan. This includes 
my body with all its parts (if one of my legs is in Buchs, then I am not in Schaan, but between it and Buchs). But it also includes my minds' activities, including my standard behaviour, etc. I am there - physically, mentally, professionally, etc.

8.3 This is our first paradigm-case of location, which was worked out by considering the half-locative - half-mereological specification of the verb "to be": to be means - to be in.

It can be summarized as follows: I am in Schaan, if each part of me is there.

Thus, according to our preliminary analysis, we can say that

I $x$ is located in $y$ means: each part of $x$ is in $y$.

8.4 Notice that location in general need not be transitive. For example, I am located in some academic institution which, in turn, is located in the network of European academies. But, clearly, $I$ am not located in this network.

Therefore none of the structures discussed previously can serve as an adequate model of location. I.e., preorders, premereologies, a fortiori mereologies, as well as Leśniewskian ontologies cannot be used to model location in general. For all of them are transitive.

8.5 The above condition I clearly has some quasi-mereological connotations. But for reasons given above and those summarized in 2.7 location can be reduced neither to mereological inclusion - for mereologies are too strong (and therefore too restrictive), nor to preorders - for they are too formal.

As a matter of fact, exactly one component of the previous explication has a clear mereological connotation, namely the notion of "a part".

8.6 The schema I can thereby be rewritten semiformally as follows:

II $\quad x$ is located in $y$ iff $\forall z(z \prec x \rightarrow z$ is in $y)$

Here $\prec$ denotes a given part-relation.

Or, in a more general way:

III $x$ is located in (i.e. is in a suitable relation to) $y$ iff $\forall z(z \prec x \rightarrow z$ is related to $y)$

Here instead of "is in" - a particular case of the ontological primitive "is" - I am using its most general form "is related to". 
8.7 In this way location is clearly connected with the part-whole relation. Indeed, for each particular type of part-whole relation $\prec$ and for a fixed variant of the primitive "is" we obtain the locative relation $L^{\prec}$ :

IV $x L^{\prec} y$ iff $\forall z(z \prec x \rightarrow z$ is related to $y)$

This suggests, for given $\prec$ and $E$, the following definition of their conjugate location

$\mathbf{V} \quad x L^{\prec} y$ iff $\forall z(z \prec x \rightarrow z E y)$

We can simplify this further by reducing the number of primitives, i.e., by defining of the part-whole relation involved in terms of the basic ontological relation $E$ after the clue of $5.1: \prec=P_{E}$.

8.8 Three remarks are in order:

(A) Sometimes we are interested in partial rather than total location, i.e., in locating by means of putting in a given connection only some important or essential or relevant parts, not all of them. This generalizes our investigation in a rather obvious way, which because of lack of space I must skip here.

(B) As the most fundamental factor of location we picked out the binary relation "to be located in". But perhaps some locations depend upon further parameters as well?

For example, we can think of location as location of something, somewhere, by somebody, in some way, etc.

But still the binary-approach seems to be basic for any further, more sophisticated treatment. Therefore hereafter I shall concentrate on it.

(C) Location is not only important and basic notion in itself. It is also useful. Among other virtues, it enables us to define several important concepts. For example, to localize means: to locate something in some area; to identify means: to locate an object and to check that it indeed is the object we are looking for; to combine means: to co-locate and to connect; to move something means: to dislocate it, to change its location, etc.

8.9 Before going on to formalities I like to extend our intuition considering a second paradigm case of location.

Imagine the following system for the voice-opening of a door: There are four words which should be said in a given order. Four cards containing 
them, plus fifth, with sequence instruction, are put into a small suitcase which, in turn, is packed into a second, bigger suitcase. Both suitcases are, of course, closed.

Now, we can truly say that the Sesam-key-system $\mathrm{S}$ is located in the first suitcase $\mathrm{S}_{1}$, which is in turn located with its content in the second suitcase $\mathrm{S}_{2}$. Indeed, each of the five parts of $\mathrm{S}$ is in $\mathrm{S}_{1}$, whereas each of the six parts of $\mathrm{S}_{2}$ (i.e. the five parts of $\mathrm{S}$ plus $\mathrm{S}_{1}$ itself) is in $\mathrm{S}_{2}$ :

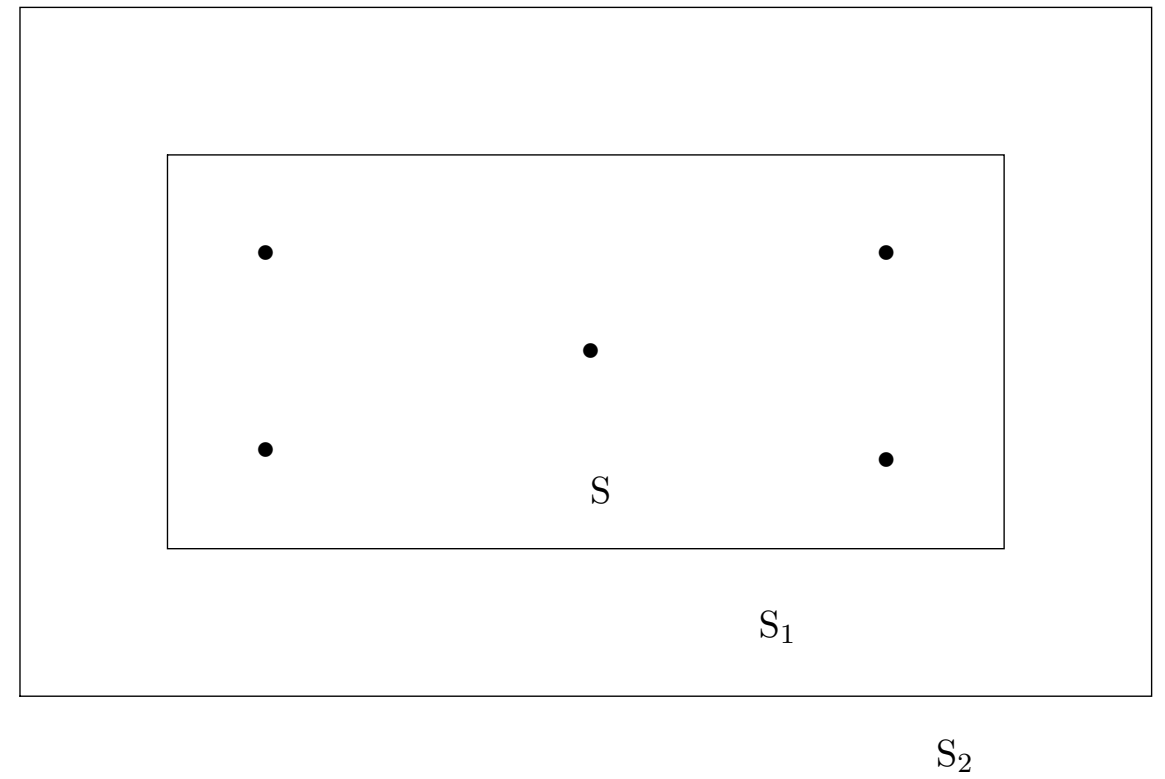

Fig. 7 .

8.10 Observe that the above locative structure clearly has two aspects: an internal or inside one - described previously, and an external or outside one - easy to see when we like to find what is located in it by opening suitcases in their proper order: starting with $\mathrm{S}_{2}$ through $\mathrm{S}_{1}$ to the final reading (decoding) of $\mathrm{S}$.

In our theory we therefore should distinguish two aspects or approaches to location: internal, which in fact was analysed in our first example, and external, which we just pointed out.

8.11 Notice that the situation described in Fig. 7 can be drawn in more 
schematic way:

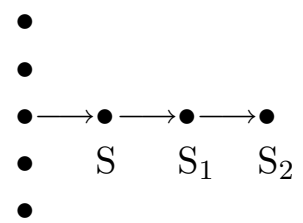

or after further simplification:

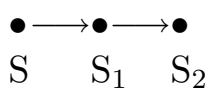

This is a typical order structure:

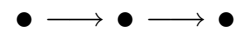

As a matter of fact, several types of orders between numbers provide, as you will see, the most useful models of location.

8.12 As a matter of fact, our second paradigmatic example of location is a version of what is probably the most famous toy-model of location: Russian dolls, called also Matrioshka, a toy made of sequences of similar babushkas (Russian country-women) one inside another.

This famous toy can, in principle, be continued up infinity, visualizing in this way the basic order-structure of natural numbers:

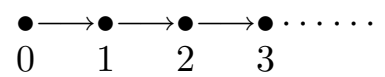

which is a locative one (for an argument cf. $\S 10.10$ ).

8.13 To resume: Our examples teach us that:

i) Location has both an internal and an external aspect. The first is approached via the part-relation, the second one - by covering relation.

ii) From the internal point of view, to be located is to be located with all parts.

iii) From the external point of view, to be located is to be covered by any locating item's cover.

iv) Location can but need not be transitive.

v) We should be ready to discuss both finite and infinite cases of location. 


\section{Fundamental Conceps of Location}

8.14 We proceed as follows: we start with a binary relation $E$ used for "is related to". This is the only primitive notion of our theory. Other notions, including order-relations, are defined.

Recall that at the beginning of ch.5 all four such relations which are $a$ priori possible were introduced, two of them $-P$ and $C$ - being preorders in general, two $-H$ and $D$ - being such for symmetric $E$ only.

Clearly, using these four part-relations we can define (after the above receipt V) at least four conjugate location-relations. In the present study, I should like to concentrate my attention on two cases: $P$-location and $C$-location (with a few of their derivativess) - for these are general, natural and amusing.

Investigation of $H$ - and $D$ - location is postponed for the occasion when symmetric variants of "is" (like those concerning identity) will call for our attention.

8.15 $P$-location is internally-oriented, for the relation $P$ goes from smaller to bigger, or from inside to outside. Call it internal location or simply location:

$$
x L y:=\forall z(z P x \rightarrow z E y), \quad \text { i.e. } P^{-1}(x) \subseteq(y]
$$

$x$ is located in $y$ iff any part of $x$ is related to $y$.

8.16 The above definition works for each case of our primitive relation $E$.

Our intuition was fixed, however, by the case "to be in". In this paradigmatic case, the above definition can be read in a very natural way: $x$ is located in $y$ iff each part of $x$ is in $y$.

By easy generalization location can, however, be defined in general, for each case of $E$, including all variants of "is".

Something similar will hold for $C$-location.

8.17 $C$-location is externally-oriented, for the cover-relation $C$ goes from outside to inside (cf. $\S 5.2$ ). $C$ is, in a sense, dual to $P: x$ is covered by $y$, if any envelope of $y$ envelops $x$ as well. Thus $C$ is externally oriented, checking if a bigger item contains a smaller one.

Call $C$-location external location, or simply allocation. Its definition is as follows:

$$
x A y:=\forall z(y C z \rightarrow x E z), \quad \text { or } C(y) \subseteq[x)
$$


$x$ is allocated in $y$ iff $x$ is related to any cover of $y$.

8.18 $P$-location and $C$-location differ in quite remarkable way.

$1^{\circ}$ Let's check, first of all, that they indeed differ. To see this consider the following

Ex. 1 Let $U=\{x, y\}, E=\{x x, x y\}$.

Hereafter, we accept the convention that the concatenation $x y$ of two objects means their ordered pair $\langle x, y\rangle$ and that in the picture of a given relation reflexive (irreflexive) points are depicted respectively by empty (nonempty) dots: $\circ$ and $\bullet$

Thus the picture of $\langle U, E\rangle$ is as follows:

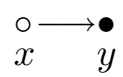

Now we can calculate that $P=\{x x, y y, x y, y x\}$, i.e. that the part-relation $P$ is full, whereas $C=\{x x, y y, x y\}$ which is not full. We should check each of eight cases involved. Consider two of them.

To see that $y P x$ we must check that for any $z$, if $z E y$ then $z E x$. Only the case $z:=x$ is relevant. But $x E x$, as required.

On the other hand, $y x \notin C$, i.e. $\neg(y C x)$. Otherwise, for any $z$, if $x E z$ then $y E z$. Take $z:=x$. Then $y E x$, for $x E x$. This, however, is not true.

Hence $P \neq C$. By a similar calculation $L=\emptyset$, whereas $A=\{x x, x y\}=$ $E$. Therefore $L \neq A$.

$2^{\circ}$ As we saw, sometimes location and allocation differ extensionally. In each case, however, they differ intensionally, stressing that we are dealing here with two opposite aspects of location.

8.19 This is stated implicitly in their definitions. For explication take a suitable example.

Consider the Russian doll in its recent version the Gorbachov's Matrioshka. This is built up in such a way that a figure of each Soviet general secretary (gensec) is contained in a figure of his successor: Lenin is in Stalin, Stalin in Malenkov, Malenkov in Khrutschov, Khrutschov in Brezhnev, Brezhnev in Andropov, Andropov in Chernenko and finally, God be praised, Chernenko in Gorbachov.

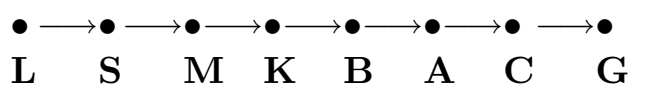


To be more formal, let $\Gamma=\{\mathbf{L}, \mathbf{S}, \mathbf{M}, \mathbf{K}, \mathbf{B}, \mathbf{A}, \mathbf{C}, \mathbf{G}\}$. Now, $E=\{\mathbf{L S}$, SM, MK, KB, BA, AC, CG .

It is easy to check that $P=\Delta_{\Gamma} \cup\{\mathbf{L} x: x \in \Gamma\}, C=\Delta_{\Gamma} \cup\{x \mathbf{G}: x \in \mathbf{G}\}$. Thus each gensec is both its own part and cover and, in addition, all gensecs have a common part: Lenin, and a common cover: Gorbachov.

To check location we should go in the "right-hand" direction, with the flow of time, from the toy's inside to its outside.

Allocation is investigated in the reverse direction, past-oriented, from the toy's outside figure - Gorbachov, by opening it successively until we reach its element - Lenin.

Using our definitions we can check that toy - location and allocation differ remarkably: $L=\{\mathbf{L S}\}, A=\{\mathbf{C G}\}$. Stalin is distinguished by location, for it is the only gensec in which something is located, namely Lenin. On the other hand, Gorbachov is the only gensec allocating something - namely Chernenko ${ }^{22}$.

8.20 The third kind of location, proper location, is defined by combination of the two previous ones:

$$
x P L y:=x L y \wedge x A y
$$

$x$ is properly located in $y$ iff it is both located and allocated in it.

8.21 To resume: Each binary relation generates its conjugate locative relations. We like to study them.

To this end, we use two part-relations: internal $(P)$ and external $(C)$. By means of them three locative relations have been defined: $L, A$ and $P L$.

In this way we obtain a net of six connected relations; $E, P, C, L, A, P L$. Their properties and interconnections form a rich and well-motivated field of formal study - the relational theory of location, or simply - locative ontology.

\section{Preliminary Observations}

8.22 I am going to present several preliminary observations. I will be rather detailed as to $L$, results can be repeated for $A$.

Remember that definitions are also axioms. Up to now, three locative axioms were introduced, namely the definitions of $L, A$ and $P L$.

\footnotetext{
${ }^{22}$ The question, is it a reasonable explanation of murdering strength of Stalin and relative disbelief of Gorbachov, is left to an ontologically oriented historiosopher.
} 
Our preliminaries concern: first, what can be deduced from the definitions alone, and next, what can be deduced from definitions in one of the traditional realms outlined in chapters $5-7$.

8.23 First of all, note that both location and allocation enjoy the following condition of well-location: what is located (allocated) is located with all its parts (allocated in all locating item's covers).

$$
x L y \leftrightarrow \forall z(z P x \rightarrow z L y), \text { and } x A y \leftrightarrow \forall z(y C z \rightarrow x A z)
$$

Proof. As regards location consider first the left-hand implication. I.e. let us suppose $\forall z(z P x \rightarrow z L y)$ and put $z:=x$. In this way we obtain $x P x \rightarrow x L y$. But $x P x$, since $P$ is reflexive; hence $x L y$.

For the converse implication assume $x L y$, i.e. $\forall z(z P x \rightarrow z E y)$, and that $z P x$. We need to prove that $z L y$, i.e., $\forall u(u P z \rightarrow u E y)$. Suppose additionally $u P z$. Using this and the second assumption we obtain $u P x$. Applying now the first assumption we obtain $u E y$, as required.

The argument in the case of allocation is analogous.

8.24 The above result deprives interest in iterating locative relations of both kinds in a simple ("natural") way. Indeed, if, for any $n \geqslant 0$, we put:

$$
\begin{array}{lll}
x L^{0} y & :=x E y & x A^{0} y \quad:=x E y \\
x L^{n+1} y & :=\forall z\left(z P x \rightarrow z L^{n} y\right) & x A^{n+1} y:=\forall z\left(y C z \rightarrow x A^{n} z\right)
\end{array}
$$

then, by (43), we immediately obtain

(44) For any $n \geqslant 1 x L^{n} y \leftrightarrow x L y$ and $x A^{n} y \leftrightarrow x A y$, i.e., $L^{n}=L$ and $A^{n}=A$.

Therefore, the only remaining cases of natural equations are: $L=E$ and $A=E$.

Are these equations true in general? No, they hold only in the locative structures which we are going to describe in the next chapter.

8.25 Location, allocation and proper location are logically stronger, i.e. more narrow in scope, than is the original relation $E$ :

$$
\begin{aligned}
& x L y \rightarrow x E y, x A y \rightarrow x E y \text { and } x P L y \rightarrow x E y, \text { i.e., } L \leqslant E, A \leqslant E \\
& \text { and } P L \leqslant E .
\end{aligned}
$$


Proof. For the first implication, recall that $x L y$ means: $\forall z(z P x \rightarrow$ $z E y)$. Take $z:=x$. By reflexivity of $P$ and by detachment we obtain the conclusion.

In the case of $A$ we reason analogously. Next, the case of $P L$ immediately follows.

8.26 We are now going to study the effect of imposing several well-known regularity conditions on our primitive relation $E$.

We start with the transitivity condition.

(46) If $E$ is transitive, then $L$ and $A$ are transitive also.

Proof. For given $x, y$ and $z$ assume $x L y$ and $y L z$. We need to prove that $x L z$, i.e., $\forall u(u P x \rightarrow u E z)$.

Enumerating our assumptions more carefully: we suppose $\forall u(u P x \rightarrow$ $u E y), \forall u(u P y \rightarrow u E z)$ and $u P x$. The first and the third assumption imply $u E y$. But $E$ is transitive, hence by (4), $E \leqslant P$. Therefore $u P y$, which together with the second assumption entails the conclusion we need: $u E z$.

The argument for $A$ is analogous.

The above implications are not reversible. To see this cf. Gorbachov's Matrioshka model of 8.19.

8.27 From (45) and (4) we immediately obtain

(47) If $E$ is transitive, then the following implications hold: $x L y \rightarrow x P y, x L y \rightarrow x C y, x A y \rightarrow x C y$ and $x A y \rightarrow x P y$.

Notice that none of the above implications is reversible, which follows by the two-element model presented in the Ex. 1 od 8.18.

Also none of these implications entail transitivity. To check such a claim we need, as usual, models. For the first and the third implication, i.e. for $L \leqslant P$ and $A \leqslant C$ see Gorbachov's matrioshka model, which satisfies both inequalities and is intransitive.

For the second implication, i.e. for $L \leqslant C$, consider the following

Ex. $2 U:=\{x, y, z\}, E:=\{x x, x y, y z\}$, i.e.

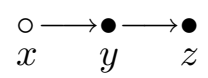

We can easily calculate that $P=\Delta \cup\{x y, y x\}, C=\Delta \cup\{x z, y z\}$, whereas $L=\emptyset$ and $A=\{y z\}$. Thus $L \leqslant C$ and $E$ is intransitive, as required. 
The remaining case can be settled by similar modelling.

8.28 Joining (4), (45) and (47) together we can write in short:

(48) For transitive $E, L \cup A \leqslant E \leqslant P \cap C$.

8.29 Turn now to the case of reflexive $E$. First notice that

(49) i) $x L x$ iff $\forall z(z P x \rightarrow z E x)$, i.e. $P^{-1}(x) \subseteq(x]$

ii) $x A x$ iff $\forall z(x C z \rightarrow x E z)$, i.e. $C(x) \subseteq[x)$.

Hence, applying (4) we obtain

$(50)$ i) $L$ is reflexive iff $E$ is reflexive

ii) $A$ is reflexive iff $E$ is reflexive.

Combining (46) with (50) we obtain also

(51) If $E$ is a preorder, then both $L$ and $A$ are preorders.

8.30 For reflexive $E$, using once again (4) and (45), we immediately obtain

$$
L \cup A \cup P \cup C \leqslant E .
$$

Notice that the five relations I have mentioned occur in two triples, for in general:

(53) $y C x \wedge x A y \rightarrow x E x$ and $y P x \wedge x L y \rightarrow y E y$.

Therefore

(54) For irreflexive $E$ : $C^{-1}$ and $P^{-1}$ are disjoint respectively with $A$ and $L: C^{-1} \cap A=\emptyset, P^{-1} \cap L=\emptyset$.

\section{Transitivity Laws}

8.31 Turn now to transitivity laws, like those which have been listed for overlapping $O$ in $\S 6.11$.

These describe connections made by superposition of two (or more) appropriate relations. If at least one of the relations involved introduces "smaller-bigger orientation", then transitivity laws are called, according to this orientation, either monotonicity or antimonotonicity laws. 
8.32 Frequently transitivity laws are the crux of important claims which sometimes seem to have little in common with the question of transitivity.

For example, it is easy to see that our first preliminary observation (43) claims, in fact, the following monotonicity for, respectively, $P$ and $L$, and $C$ and $A$ :

$$
z P x \wedge x L y \rightarrow z L y, \text { and } x A y \wedge y C z \rightarrow x A z .
$$

The first claim is the law of left-monotonicity for location: Any part of what is located is located in the same item, i.e., location is location with parts; whereas the second claim is the law of right-hand monotonicity for allocation: allocation means allocation in allocating item's covers.

8.33 Notice that the two accompanying laws: the right-monotonicity law for location and the left-monotonicity law for allocation hold in general as well:

(56) $x L y \wedge y P z \rightarrow x L z$ and $x C y \wedge y A z \rightarrow x A z$.

8.34 Hence, using also (47) and (55) we obtain

$$
\begin{aligned}
& z P x \wedge x L y \rightarrow z E y, x A y \wedge y C z \rightarrow x E z, x L y \wedge y P z \rightarrow x E z \text { and } \\
& x C y \wedge y A z \rightarrow x E z .
\end{aligned}
$$

8.35 By (45) we know that in general $L \leqslant E$ and $A \leqslant E$. But $P$ and $C$ need not be comparable with $E$. They can be bigger, smaller or even cross with the relation $E$.

However, by (55)-(57), we obtain that superposition of $P$ and $C$ respectively with $L$ and $C$ are in each case smaller than $E$.

$$
P \circ L \leqslant L \leqslant E, L \circ P \leqslant L \leqslant E, A \circ C \leqslant A \leqslant E \text { and } C \circ A \leqslant A \leqslant E .
$$

8.36 But $P$ and $C$ are reflexive. Hence for any binary relation $R$ defined on their universe $U: R \leqslant R \circ P, R \leqslant P \circ R, R \leqslant R \circ C$ and $R \leqslant C \circ R$.

Therefore, (58) entails

$$
P \circ L=L=L \circ P \text { and } C \circ A=A=A \circ C \text {. }
$$

8.37 Consider now the problem of left and right monotonicity for $P$ and $C$ with respect to the original relation $E$.

Two of the four a priori given statements hold in general: the right-monotonicity for $P$ and the left-monotonicity for $C$. Indeed 
(60) $x E y \wedge y P z \rightarrow x E z$ and $x C y \wedge y E z \rightarrow x E z$;

If $x$ is in $y$ and $y$ is a part of $z$ then $x$ is in $y$, and if $x$ is

covered by an object which is in $z$ then $x$ is in $z$.

The remaining two laws hold under a special proviso, for example, for preorders:

(61) If $E$ is a preorder, then $x P y \wedge y E z \rightarrow x E z$ and $x E y \wedge y C z \rightarrow x E z$. I.e., if $x$ is a part of something which is in $z$ then $x$ is in $z$ as well, and if $x$ is in something which is covered by $z$ then $x$ is in $z$.

8.38 Arguing in the same way as before we can justify an algebraic version of the last two claims:

(62) In general: $E \circ P=E$ and $C \circ E=E$, whereas under the proviso that $E$ is a preorder relation: $P \circ E=E$ and $E \circ C=E$.

8.39 Passing to monotonicity laws for two locative-relations which interest us especially, I will list two families of laws: the first for transitive $E$, the second for preorders

(63) Let $E$ be transitive. Then $x E y \wedge y L z \rightarrow x E z, \quad x E y \wedge y A z \rightarrow x E z$ and $x L y \wedge y E z \rightarrow x L z$. Informal reading: If $x$ is in something which is located in $z$ then $x$ is in $z$, if $x$ is in something which is allocated in $z$ then $x$ is in $z$, and whatever is located in a case of $z$ is located in $z$ itself.

Proof. All cases follow immediately from the definitions.

Consider, for example, the last implication. To this end assume that $y E z$ and $x L y$, i.e., for any $w, w P x \rightarrow w E y$. In addition we assume the supposition of $x L z$, i.e., that for a given $u, u P x$. By particularization of the second assumption we obtain: $u E y$. This, by the first assumption and transitivity of $E$ implies that $u E z$. Therefore $x L z$, as required.

(64) If $E$ is a preorder relation, then $x E y \wedge y L z \rightarrow x L z, x E y \wedge y A z \rightarrow$ $x A z$ and $x A y \wedge y E z \rightarrow x A z$. Speaking informally: If $x$ is in something which is located (allocated) in $z$, then $x$ is in $z$; and whatever is allocated in a case of $z$ is allocated in $z$ itself.

The proof is similar to the last one.

Finally, observe that none of the above six laws is true in general. As a matter of fact, they are weaker than their assumptions.

8.40 Expressing the above laws in terms of superposition we have 
(65) i) If $E$ is transitive, then $E \circ L \leqslant E, E \circ A \leqslant E$ and $L \circ E \leqslant L$

ii) If $E$ is a preorder, then $E \circ L \leqslant L, E \circ A \leqslant A$ and $A \circ E \leqslant A$.

8.41 To conclude, transitivity laws are indeed the most natural laws comparing the 5 relations under investigation: $E, P, C, L$ and $A$. A priori there is quite a $\operatorname{lot}^{23}$ of such laws, 19 of which we have considered explicitly: the classical transitivity law for $E$ - in chapters 4 and 5, and 18 laws discussed in this subchapter. Because of limitations of space, an investigation of the further cases is left for another occasion.

\section{LOCATIVE ONTOLOGIES}

\section{Axioms}

9.1 Now we are passing to a rather subtle question of axiomatization of locative structures. Which axioms should be added to obtain a more adequate formalisation of the relational concept of location?

This is both a formal and an essential question. We need axioms both well-motivated and rich in consequences. For axioms are judged by their fruits.

9.2 First observe that we can accept as axiom any well-motivated law of locative transitivity which is not generally valid.

As we just learned, there are plenty of candidates. I will discuss them later on.

9.3 Consider now the following way of finding axioms:

Suppose we are interested in the question: How many? I.e., for a given relational universe $\langle U, E\rangle$ and for its locative relations $L$ and $A$, we would like to know how many items are located (allocated) one in another?

The extreme positions we can consider are as follows:

The strongest one, anything is located (allocated) in everything:
FUL $\forall x \forall y x L y$
full location,
FUA $\forall x \forall y x A y$
- full allocation.

\footnotetext{
${ }^{23}$ Calculation of the number of such laws depends on further assumptions, cf. forthcoming remarks concerning relational syllogistic.
} 
It is easy to see that these axioms are so strong that they axiomatize full structures:

(66) FUL or FUA holds in $\langle U, E\rangle$ iff $E$ is full, i.e., $\forall x \forall y x E y$.

Hence, both candidates, being too strong are uninteresting.

The opposite view that a relational structure has no case of location (allocation): $\neg \exists x \exists y x L y$ or $\neg \exists x \exists y x A y$ is also pathological, hence uninteresting.

9.4 The next assumptions of this type are assumptions of nonemptiness of locative relations:

NEL $\exists x \exists y x L y$

NEA $\exists x \exists y x A y$

These are more interesting conditions. Clearly, both NEL and NEA hold only in nonempty structures, i.e., they imply that $E \neq \emptyset$.

Query: Find suitable conditions to reverse the above implications. Characterize both NEL and NEA.

9.5 Turn to our fundamental question which generates the set of basic axioms concerning location.

Recall that we investigate interconnections between the following five relations: the original relation $E$ which was introduced to formalize the verb "to be"; its two conjugate preorders $P$ and $C$ which, in the realm of $E$, formalize two basic part-relations, respectively "to be a part of" and "to be covered by"; and its two conjugate locative relations: internal $-L$ and external $-A$.

The basic problem of the theory of location is to compare these five relations.

By now we know that in general $L \leqslant E$ and $A \leqslant E$ and we know cases in which $E \leqslant P$ and $E \leqslant C$ (for transitive $E$ ), $P \leqslant E$ and $C \leqslant E$ (for reflexive $E$ ), and $P=E=C$ (for preorders).

The remaining cases, however, are still obscure.

The main family of our axioms is introduced to answer those questions of comparison.

9.6 The basic question of this sort is: In which case is the starting relation $E$ itself locative? In other words, characterize structures in which "to be related" equals "to be located in". 
Previously two types of location were distinguished: internal $(L)$ and external $(A)$. Having this in mind we are ready to list the four a priori possible positive answers to our question.

The relational universe $\langle U, E\rangle$ is said to be:

Internally locative iff it satisfies the following axiom:

INL

$$
E=L,
$$

i.e., if "to be related" is "to be located";

Externally locative iff it satisfies the following axiom:

\section{EXL}

$$
E=A,
$$

i.e., if "to be related" is "to be allocated";

Locative iff it is both internally and externally located, i.e., it satisfies

$\mathbf{L}$

$$
L=E=A
$$

i.e., "to be related" is "to be both located and allocated";

Weakly locative iff it is either internally or externally locative or both, i.e., it satisfies:

WL

$$
E=L \vee E=A \text {. }
$$

Notice that the convention A of $\S 4.11$ has introduced other names for the above axioms: EL for INL, EA for EXL, and LEA for L.

9.7 Locative ontologies of appropriate kinds are structures satisfying suitable axioms from the above list.

Observe that locative ontologies are axiomatized according to a general recipe used, with some success, several times before: For given relations consider cases where their graphs collapse, i.e., they are set-theoretically (or extensionally) equal.

9.8 The above four axioms constitute our first, fundamental set of axioms for location.

Two other sets are connected with comparison of $L$ with $P, A$ with $C$, and $L$ with $A$. 
9.9 It is easy to see that $L$ and $P$ as well as $A$ and $C$ are, in general, mutually incomparable.

Outside of the realm of preorders location means something other than "to be a part of".

To see this consider the following model:

Ex. 3 It contains two pieces which mirror each other, i.e., everything which is in the first is in the second and conversely. In such a case we can say that the first item is in the second and, conversely, the second is in the first.

In other words, our model constitutes an elementary loop:

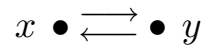

Here $U:=\{x, y\}$ and $E:=\{x y, y x\}$. Now it is easy to calculate that $P=\{x x, y y\}=C$, whereas $L=\{x y, y x\}=E=A$.

Hence our loop satisfies all axioms of the first group. Therefore, it is a locative structure.

But its part-relations are strongly incomparable with suitable locative relations, for they are mutually disjoint with them: $P \cap L=\emptyset$ and $C \cap A=\emptyset$.

Comparison of part- and locative-relations is therefore an essential question. It fully deserve to be decided by axioms.

9.10 By the way, notice that the above reasoning justifies the following useful observation:

(67) If $P$ and $C$ are the smallest possible, i.e. if $P=\Delta=C$ then the structure $\langle U, E\rangle$ is locative, i.e., it fulfils $L=E=A$.

For, under our assumption, $\forall z(z P x \rightarrow z E y)$ is equivalent to $x E y$, hence $x L y \leftrightarrow x E y$. Analogously for $A=E$.

Observe that instead of $P_{E}=\Delta$ we can assume $C_{E}=\Delta$, because

$$
P_{E}=\Delta \quad \text { iff } \quad C_{E}=\Delta .
$$

Cf. $§ 9.34$ below.

9.11 The second family of locative comparison axioms is as follows:

$\begin{array}{llll}\boldsymbol{L P} & L \leqslant P & \boldsymbol{A C} & A \leqslant C \\ \boldsymbol{P L} & P \leqslant L & \boldsymbol{C A} & C \leqslant A \\ \mathbf{L P} & L=P & \mathbf{A C} & A=C\end{array}$


and for the case of strong incomparability:
$\operatorname{LDSP} L \cap P=\emptyset$
$\operatorname{ADSC} A \cap C=\emptyset$

The meaning of the axiom $\mathbf{L P}$ is clear: location and parthood relations coincide. To emphasize an implicit connection with preorders (cf. (114) in $\S 10.18)$ the axiom LP is called the axiom od prelocation. Similarly, AC is the axiom of preallocation.

9.12 The third and final group of comparison axioms concerns comparison of the two locative relations, $L$ with $A$.

By Gorbachov's Matrioshka model we know that in general $L$ and $A$ are incomparable, for there $L \cap A=\emptyset$ (cf. 8.19).

Hence any comparison is essential. The four axioms below exhaust all regular situations:
LA $\quad L \leqslant A$
$\boldsymbol{A L} \quad A \leqslant L$
LA $L=A$
$\operatorname{LDSA} \quad L \cap A=\emptyset$

9.13 The main body of locative ontology is yielded by investigations of the consequences of several groups of the above axioms.

\section{Immediate Consequences of the Axioms}

9.14 Inquiry into consequences of locative axioms enumerated previously is a rather complex exercise. Therefore it needs some organization.

I will try to be rather scrupulous as to the proper axioms of location from the first group, being much more sketchy on the others.

The order of my discussion is as follows: I will start with immediate reformulations and consequences of the axioms under investigation, passing next to several indirect consequences. In subsequent parts of the paper I will try to clarify further the structure of the realm of locative ontologies. Finally, I will discuss several applications of locative ontology, including philosophical ones.

9.15 To throw light on the meaning of the basic locative axioms INL, EXL and $\mathbf{L}$ the following definitions are introduced: 
For a given relation $E$ on its universe $U$ an object $x$ is well-locating (well-allocating) iff all its subobjects are located (allocated) in it:

$$
\begin{aligned}
& W L(x):=\forall y(y E x \rightarrow y L x) \\
& W A(x):=\forall y(y E x \rightarrow y A x) .
\end{aligned}
$$

For symmetry's sake, $x$ is said to be well-located (well-allocated) iff any of its overobject locates (allocates) it:

$$
\begin{aligned}
& L W(x):=\forall y(x E y \rightarrow x L y) \\
& A W(x):=\forall y(x E y \rightarrow x A y) .
\end{aligned}
$$

In quite a lot of cases both locating (allocating) and locative (allocative) items occur quite often. Also, in quite a lot of universes they play a quite noticable role. Therefore it is an useful exercise to characterize well-locating (allocating) and well-located (allocated) objects of a given relational structure.

Now we see the meaning of our axioms. The axiom INL picks spaces in which each object is both well-located and well-locating. Similarly, the axiom EXL distinguishes spaces in which each object is both well-allocated and well-allocating, whereas the axiom $\mathbf{L}$ says the same both for location and allocation.

9.16 Both axioms of internal and external location have quite a lot of illuminating equivalents.

Consider first INL. In its developed form it is

\section{INL1}

$$
x E y \leftrightarrow \forall z(z P x \rightarrow z E y)
$$

I.e., it exactly expresses the idea of location through $E$ by means of $P$. To see this, recall that the relation $E$ is the only primitive notion of the theory of location. $P$ is defined by it in such a way that it really captures essential features of the relation "to be a part of". Hence the axiom INL1 says: to be related is to be related with all parts, but this means: to be fully located.

9.17 In turn, by elimination of $P$ we obtain the explicit form of INL which is an implicit "axiomatic" definition of the primitive $E$ in the theory of internal location:

INL2

$$
x E y \leftrightarrow \forall z[\forall u(u E z \rightarrow u E x) \rightarrow z E y]
$$


Extracting quantifiers we obtain the prenex-form of the axiom

INL3

$$
\forall x \forall y \forall z \exists u[x E y \rightarrow((u E z \rightarrow u E x) \rightarrow z E y)]
$$

Hence, in spite of its apparent clarity, the axiom of internal location has a rather strong logical form. It is a first order formula of the type $\forall^{3} \exists$, hence implicitely it is an existential formula.

9.18 In the light of (45) we immediately obtain

(69) INL is equivalent to $\boldsymbol{E L}: E \leqslant L$.

Indeed, $\boldsymbol{E} \boldsymbol{L}$ is expressed by

INL4

$$
x E y \rightarrow \forall z(z P x \rightarrow z E y)
$$

which, by (45), is equivalent to INL.

9.19 Moreover, the last formula is exactly the expression of the first monotonicity law stated in (61) for preorders. Hence

$$
\text { INL iff } z P x \wedge x E y \rightarrow z E y
$$

which, in turn, enables us to obtain the following generalization of one of the facts stated in (62):

(71) INL iff $P \circ E=E$.

In this way facts which were previously stated for preorders can be generalized to, what will be proved, the broader domain of locative structures.

To conclude, some transitivity laws which are not generally true occur to be very natural axioms.

9.20 Observe that

$$
E \leqslant P \text { iff } E \leqslant C \text {, and } P \leqslant E \text { iff } P \leqslant C .
$$

Indeed, both sides of the first equivalence are, by (4), equivalent to transitivity of $E$; whereas both sides of the second condition are equivalent to $E$ reflexivity. Conditions compared in (72) must therefore be equivalent.

This, however, does not mean that $P$ and $C$ are in all cases comparable. This regularity is achieved only in the case of locative ontologies.

9.21 In fact, internally locative structures are exactly those which make $P$ weaker than $C$ : 
(73) INL iff $\boldsymbol{P C}: P \leqslant C$.

Proof. By (69) we need instead of INL to consider only its right-hand implication: $x E y \rightarrow x L y$, which is equivalent to INL4: $x E y \rightarrow \forall z(z P x \rightarrow$ $z E y$ ), which in sequence is logically equivalent to an open formula $x E y \rightarrow$ $(z P x \rightarrow z E y)$. This, in turn, is equivalent to $z P x \rightarrow(x E y \rightarrow z E y)$, which is again equivalent to $z P x \rightarrow \forall y(x E y \rightarrow z E y)$. This, finally, is equivalent to $z P x \rightarrow z C x$, i.e., $P \leqslant C$.

As regards the logic behind (73), the crux of the above proof lies in the free use of the exportation laws of classical quantifier logic, made possible by the very special distribution of variables in the starting formula.

9.22 Equivalents of EXL are, mutatis mutandis, similar to those for INL. Also, they can be proved by quite analogous arguments.

Therefore I can here only enumerate them, leaving proofs to the reader himself.

$$
\begin{aligned}
& \text { EXL } \quad \text { is, in turn, equivalent to: } \\
& \text { EXL1 } x E y \leftrightarrow \forall z(y C z \rightarrow x E z), \\
& \text { EXL2 } x E y \leftrightarrow \forall z[\forall u(z E u \rightarrow y E u) \rightarrow x E z], \\
& \text { EXL3 } \forall x \forall y \forall z \exists u[x E y \rightarrow((z E u \rightarrow y E u) \rightarrow x E z], \text { and } \\
& \text { EXL4 } x E y \rightarrow \forall z(y C z \rightarrow x E z) .
\end{aligned}
$$

Hence, the axiom EXL has the same logical complexity as INL. It is an $\forall^{3} \exists$-formula. Therefore, it is in fact an existential axiom.

9.23 Next, EXL is equivalent to the second monotonicity law from (61):

EXL iff $x E y \wedge y C z \rightarrow x E z$

which, in turn, implies the following generalization of the second part of (62):

(76) EXL iff $E \circ C=E$.

On the other hand,

(77) EXL is equivalent to $\boldsymbol{C P}: C \leqslant P$.

9.24 The strong location axiom $\mathbf{L}$ is, by definition, the conjunction of INL and EXL. It says that $L=E=A$, which can be simplified as follows:

$\mathbf{L}$ iff $E=L \cap A$.

Further equivalents of $\mathbf{L}$ can be obtained by appropriate conjunctions of equivalents of INL and EXL. 
(79) The following are equivalents of $\mathbf{L}$ :

i) Both $z P x \wedge x E y \rightarrow z E y$ and $x E y \wedge y C z \rightarrow x E z$ hold,

ii) $P \circ E=E=E \circ C$

iii) PC: $P=C$.

Then we see that in the proper or strong locative structures both internal and external part-relation coincide. Their difference is, in a sense, the cause of the difference between two locative relations.

9.25 Clearly, equivalents of the weak axiom of location WL are disjunctions of appropriate conditions for internal and external location. Hence

(80) WL iff $E=L \cup A$.

9.26 The logical dependencies between locative axioms of the first group can be summarized in the following diagram:

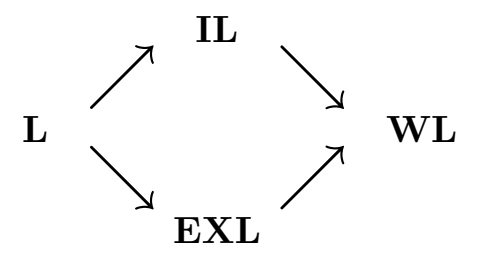

Fig. 8.

That the implications indicated by the arrows hold is clear. However, claims that no arrow is reversible and that INL and EXL are independent need suitable models. They will be provided in one of the subsequent subchapters, which is especially devoted to models.

9.27 Now we are going to discuss very briefly the second group of locative axioms, those listed in $\S 9.11$.

First of all, note that two of them are connected with reflexivity: $\boldsymbol{P L}$, as well as $\boldsymbol{C A}$, axiomatizes the well-known domain of reflexive orders:

$(81)$ i) $P \leqslant L$ iff $P \leqslant E$ iff $E$ is reflexive

ii) $C \leqslant A$ iff $C \leqslant E$ iff $E$ is reflexive.

Proof. The second equivalence, both in i) and ii) has been stated previously (cf. $\S 5.5$, claim (4)).

To prove the left-hand implication of the first equivalence in i) assume $P \leqslant E$. We need to check that for any $x$ and $y$, if $x P y$ then $x L y$, i.e., that 
$x P y \rightarrow \forall z(z P x \rightarrow z E y)$. Thus we can, in addition, assume $x P y$ and $z P x$ with the aim deducing $z E y$. From the second and the third assumption, by transitivity of $P$, we obtain $z P y$, which, by the first assumption, implies $z E y$, as required.

The right-hand implication is immediate, for in general $L \leqslant E$ (cf. (45)), hence $P \leqslant L$ does indeed imply $P \leqslant E$.

The remaining case of the first equivalence from ii) can be checked in a similar way.

9.28 The other two axioms, namely $\boldsymbol{L P}$ and $\boldsymbol{A} \boldsymbol{C}$, are connected with transitivity, but not in so strict a way as the two previous ones.

(82) If $E$ is transitive, then $L \leqslant P$ and $A \leqslant C$ but not conversely:

$L \leqslant P \wedge A \leqslant C$ does not imply the transitivity of $E$.

Proof. The first claim immediately follows from (4) and (45). Indeed, if $E$ is transitive then, by (4), $E \leqslant P$. On the other hand, by (45), $L \leqslant E$. Hence $L \leqslant P$. Similarly for $\boldsymbol{A} \boldsymbol{C}$.

For the second claim recall Gorbachov's Matrioshka model, which satisfies both $\boldsymbol{L P}$ and $\boldsymbol{A} \boldsymbol{C}$ but is intransitive.

9.29 In conclusion:

(83) If $E$ is a preorder, then $L=P$ and $A=C$.

The above implication is not reversible. Indeed:

(84) The strongest combination of axioms from the second group, i.e. the conjunction $L=P \wedge A=C$, does not imply that $E$ is transitive.

To see this consider the following

Ex. 4 Let $U=\{x, y, z\}$ and $E=\{x x, y y, z z, x y, y z\}$. I.e.

$$
\stackrel{\circ \longrightarrow}{x} \mathrm{O} \longrightarrow \mathrm{O}
$$

It is easy to calculate that $P=\Delta \cup\{x y\}=L$ and $C=\Delta \cup\{y z\}=A$, but $E$ is transparently non-transitive.

Combining this observation with the two previous ones we obtain that the axioms under consideration: $L=P, A=C$ and $L=P \wedge A=C$ are strictly intermediate between reflexivity and preordering. Indeed, each of 
them is weaker than reflexivity plus transitivity but stronger than reflexivity alone.

9.30 Observe that $\boldsymbol{L P}$ and $\boldsymbol{A C}$ are, like INL and EXL, semiexistential formulas. Indeed

(85) $\quad \boldsymbol{L P}$ is equivalent to: $\forall x \forall y \exists z((z P x \rightarrow z E y) \rightarrow x P y)$; whereas $\boldsymbol{A C}$ is equivalent to: $\forall x \forall y \exists z((y C z \rightarrow x E z) \rightarrow x C y)$.

9.31 Turn now to the remaining disjointness axioms LDSP and ADSC. We will pay attention only to the first axiom: $L \cap P=\emptyset$, all the observations stated below can automatically be extended to the second one.

First, observe that disjointness of $L$ with $P$ implies that $L$ is irreflexive.

(86) $L \cap P=\emptyset \rightarrow L$ is irreflexive.

Indeed, by (2), $\Delta \subseteq P$. But $P \cap L=\emptyset$, hence $\Delta \cap L=\emptyset$. Applying now (1) we reach the desired conclusion.

Next observe that LDSP with $E$-transitivity implies that $L$ is empty.

(87) LDSP and $E$ transitive $\rightarrow L=\emptyset$.

Proof. Take a transitive relation $E$. By (4) and (45) we have: $L \leqslant E \leqslant$ $P$. Hence $L \cap P=L$. But, by our first assumption, $L \cap P=\emptyset$. Therefore $L=\emptyset$, as required.

On the other hand, by (50) and (86) we obtain:

(88) $L \cap P=\emptyset$ implies that $E$ is not reflexive.

Combining the last two statements together we have:

(89) If $L$ is nonempty and LDSP, then $E$ is neither reflexive nor transitive.

9.32 Finally consider the question of comparison of the two locative relations, $L$ with $A$, which is settled by the axioms from the third group.

These axioms are clearly connected with the proper location axioms from the first group. Indeed

(90) L implies LA.

But not conversely. To see this, consider 
Ex. 5 Let $U=\{x, y, z, u\}$ and $E=\{x z, y u\}$. I.e.

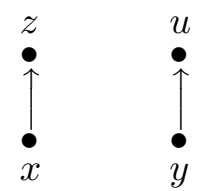

Here $L=\emptyset=A$, i.e $\mathbf{L A}$ holds, but $\mathbf{L}$ is not valid, as $L, A<E$.

On the other hand

(91) $\quad \boldsymbol{L} \boldsymbol{A} \wedge$ INL $\rightarrow$ EXL, $\boldsymbol{A L} \wedge$ EXL $\rightarrow$ INL

A fortiori:

(92) If $L$ equals $A$, i.e. $\mathbf{L A}$, then $\langle U, E\rangle$ is internally locative iff it is externally locative,i.e., INL $\leftrightarrow$ EXL.

The implications from (91) and (92) are also not reversible.

9.33 To finish our brief discussion of the last group of locative axioms, notice that they can be combined with the axioms of the second group to introduce further regularities, similar to those discussed in the previous section.

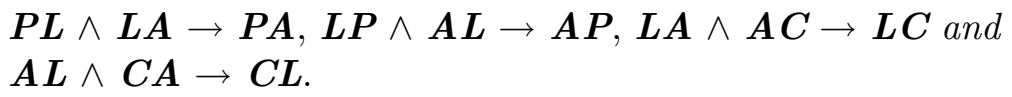

\section{Duality}

9.34 We know that the four derivative relations under investigation: $P$, $C, L$ and $A$ in general differ both extensionally and intensionally. However, when taken in respective pairs: $P$ with $C$ and $L$ with $A$, they are clearly connected. They, in a sense, mirror each other.

As a matter of fact, they are converse-dual. For a given relation $E$, its part-relation $P_{E}$ can be considered as the converse of the covering relation taken for the relation $E^{-1}$. On the other hand, the $E$-covering $C_{E}$ is the converse of the part relation for $E^{-1}$. Moreover, $E$-location equals the converse of $E^{-1}$ allocation and vice versa. In symbols

$$
P_{E}=\left(C_{E^{-1}}\right)^{-1}, C_{E}=\left(P_{E^{-1}}\right)^{-1}, L_{E}=\left(A_{E^{-1}}\right)^{-1} \text { and } A_{E}=\left(L_{E^{-1}}\right)^{-1}
$$

which immediately follows from 


$$
\begin{aligned}
& \left(P_{E}\right)^{-1}=C_{E^{-1}},\left(C_{E}\right)^{-1}=P_{E^{-1}},\left(L_{E}\right)^{-1}=A_{E^{-1}} \text { and } \\
& \left(A_{E}\right)^{-1}=L_{E^{-1}} .
\end{aligned}
$$

Proof. Observe first that by the well known laws of the relation calculus: $\left(R^{-1}\right)^{-1}=R$ and $R=S$ iff $R^{-1}=S^{-1}$, hence (94) indeed follows from (95).

To prove (95) consider first the case of $P_{E}$. Let $x\left(P_{E}\right)^{-1} y$, i.e., $y P_{E} x$. By definition of $P_{E}$ it is equivalent to $\forall z(z E y \rightarrow z E x)$ which, in turn, is equivalent to: $\forall z\left(y E^{-1} z \rightarrow x E^{-1} z\right)$, i.e. to $x C_{E^{-1}} y$.

Consider now the case of $L_{E}$. Proceeding similarly, $x\left(L_{E}\right)^{-1} y \leftrightarrow y L_{E} x \leftrightarrow$ $\forall z\left(z P_{E} y \rightarrow z E x\right)$, which, by the previous case, is equivalent to: $\forall z\left(z\left(C_{E}\right)^{-1} y\right.$ $\rightarrow z E x)$ which, in turn, is equivalent to: $\forall z\left(y C_{E^{-1}} z \rightarrow x E^{-1} z\right)$, hence to: $x A_{E^{-1}} y$.

The remaining two cases can be checked analogously.

9.35 As immediate corollaries we have

(96) $L_{E}=P_{E}$ iff $A_{E^{-1}}=C_{E^{-1}}, A_{E}=C_{E}$ iff $L_{E^{-1}}=P_{E^{-1}}$, $L_{E}=E$ iff $A_{E^{-1}}=E^{-1}$, and $L_{E}=A_{E}$ iff $L_{E^{-1}}=A_{E^{-1}}$.

(97) If $E$ is symmetric, then to be a part of is the converse to be covered by and conversely. Moreover, location is the converse of allocation and vice versa: $P=C^{-1}, C=P^{-1}, L=A^{-1}$ and $A=L^{-1}$.

Indeed, by (1), $E$ is symmetric iff $E=E^{-1}$. Hence, under the proviso of the symmetry of $E$ we can rewrite the equations in (94) in a form depending only on $E$. This is exactly what we need, for by our starting convention subscripts can be omitted, if unnecessary.

9.36 To conclude: By the duality of $P$ with $C$ and $L$ with $A$ we can reduce the number of notions of our theory. Instead of five: a primitive $E$ and the four derivatives - $P, C, L$ and $A$ we can think of our subject as concerning interconnections between the original relation $E$ and the three derivatives: converse-relation, part-relation and location with principles saying that internal location equals the converse of external location taken for the converse of the starting relation, etc.

However, in this way no essential reduction is in fact achieved, but only further clarification of the interconnections between parts and covers as well as between location and allocation. 


\section{Axioms Revisited}

9.37 It is illuminating, in light of the previous discussion, to see our axioms once again.

The first group form the following axioms concerning location:

INL $L_{E}=E$

EXL $\quad L_{E^{-1}}=E^{-1}$, hence

L $\quad L_{E}=E$ and $L_{E^{-1}}=E^{-1}$.

In short, the proper axioms of location say respectively that the primitive relation $E$ is locative, that its converse is locative, and that both the relation and its converse are locative.

The strongest axioms from the second group say respectively that

LP $\quad L_{E}=P_{E}$

AC $\quad L_{E^{-1}}=P_{E^{-1}}$.

I.e., that location as determined by the starting relation $E$ equals to its parthood relation, and that location determined by the converse relation $E^{-1}$ equals to its parthood relation.

Finally, the axiom $\mathbf{L A}$ is equivalent to: $\left(L_{E}\right)^{-1}=L_{E^{-1}}$. In plain words, the converse of location equals location determined by the converse.

9.38 To conclude: You can, if you like, replace the two-aspect, $P-C$ and $L-A$, description of $a$ given relational domain $\langle U, E\rangle$ by one-aspect, $P-L$, description of two domains: $\langle U, E\rangle$ and $\left\langle U, E^{-1}\right\rangle$.

Both approaches are indeed equivalent.

\section{Preservation}

9.39 Notice that the above observations, in particular (96), can be expressed also in the form of the following preservation theorems:

i) $\langle U, E\rangle$ is internally locative iff $\left\langle U, E^{-1}\right\rangle$ is externally locative, i.e., the relational frame verifies INL iff its converse verifies $\mathbf{E X L}$.

In symbols: $\langle U, E\rangle \models \mathbf{I N L}$ iff $\left\langle U, E^{-1}\right\rangle \models \mathbf{E X L}$;

ii) $\langle U, E\rangle$ is externally locating iff $\left\langle U, E^{-1}\right\rangle$ is internally locative, i.e., $\langle U, E\rangle \models$ EXL iff $\left\langle U, E^{-1}\right\rangle \models$ INL;

iii) $\langle U, E\rangle$ is locative iff $\left\langle U, E^{-1}\right\rangle$ is locative, i.e., $\langle U, E\rangle \models \mathbf{L}$ iff $\left\langle U, E^{-1}\right\rangle \models \mathbf{L}$. 
In plain words: the taking of converses preserves proper location.

Similar statements hold also for the remaining two groups of axioms:

$(99) \quad$ i) $\langle U, E\rangle \models \boldsymbol{L P}$ iff $\left\langle U, E^{-1}\right\rangle \models \boldsymbol{A C}$

ii) $\langle U, E\rangle \models \boldsymbol{P L}$ iff $\left\langle U, E^{-1}\right\rangle \models \boldsymbol{C A}$

iii) $\langle U, E\rangle \models \mathbf{L P}$ iff $\left\langle U, E^{-1}\right\rangle \models \mathbf{A C}$

$(100) \quad$ i) $\langle U, E\rangle \models \boldsymbol{L} \boldsymbol{A}$ iff $\left\langle U, E^{-1}\right\rangle \models \boldsymbol{A} \boldsymbol{L}$

ii) $\langle U, E\rangle \models \boldsymbol{A} \boldsymbol{L}$ iff $\left\langle U, E^{-1}\right\rangle \models \boldsymbol{L} \boldsymbol{A}$

iii) $\langle U, E\rangle \models \mathbf{L A}$ iff $\left\langle U, E^{-1}\right\rangle \models \mathbf{L} \mathbf{A}$ 


\section{PART III. LOCATIVE ONTO-LOGIC}

\section{PREORDERS AND LOCATIVE STRUCTURES}

\section{Generalia}

10.1 We are going to provide a rather detailed comparison of locative structures with preorders. As the reader will see, locative structures generalize preorders.

The realm of locative structures is much broader in scope than the realm of preorders. Fortunately, locative structures enjoy a great wealth of the regularities of preorders. To make this clear, in what follows I am going to study rather carefully the counterparts of the basic features of preorders, which were brought together in chapters $4-6$ above.

10.2 First of all, observe that each of the three types of location studied here is weaker than preordering.

This has already been established in (83) for locative structures fulfilling axioms from the second group: If $E$ is a preorder relation, then $\langle U, E\rangle$ satisfies both $L=P$ and $A=C$.

Similarly for the remaining two families of axioms:

(101) If $\langle U, E\rangle$ is a preorder relation, then $L=E=A$, i.e., $\langle U, E\rangle$ is locative.

Proof. Assume that $E$ is reflexive and transitive as well as the assumptions of $E \leqslant L$ : for any $x$ and $y, x E y$ and $z P x$. By the last assumption: $\forall u(u E z \rightarrow u E x)$, hence $z E z \rightarrow z E x$. But $E$ is reflexive, therefore $z E x$. Applying now transitivity of $E$, we obtain that $z E y$, as required.

The remaining inequality: $E \leqslant A$ can be checked analogously.

$$
\text { If } E \text { is a preorder relation, then } L=A \text {; i.e. }\langle U, E\rangle \models \mathbf{L A} \text {. }
$$

This follows immediately by (83) and (101). 
10.3 To resume:

(103) For preorders all five relations under consideration: $E, P, C, L$ and $A$ coincide, i.e., $L=P=E=C=A$.

From the point of view of locative ontology preorders are therefore very regular, hence strong, structures.

Too regular, however. Recall that the distinction between $P$ and $C$ as well as between $L$ and $A$ discriminates two aspects of the parthood and the locative relations generated by the starting relation $E$. As we saw several times before, this distinction is important.

Yet it disappears in all preorders. Therefore, for a more discriminative study of location, preorders, a fortiori mereologies, are too powerful and too regular. In the realm of preorders we lose even the distinction between "to be a part of" and "to be located in".

10.4 Anyway, preorders are rich in nice equations. In addition to those enumerated in (103) let me list here those equations studied previously which hold in the realm of preorders:

$P_{E}=E, P_{E^{-1}}=E^{-1},\left(P_{E}\right)^{-1}=P_{E^{-1}}, L_{E}=E, L_{E^{-1}}=E^{-1},\left(L_{E}\right)^{-1}=$ $L_{E^{-1}}$ plus the analogous equations for $C_{E}, C_{E^{-1}}, A_{E}$ and $A_{E^{-1}}$.

\section{Comparison}

10.5 To what extent locative structures of the three kinds introduced previously in chapter 9 enrich our usual universe of discourse?

To answer this question we are going to provide a systematic comparison of the realms of the different locative structures with the realms studied previously: mereologies, premereologies, preorders and Leśniewski's ontologies (cf. Figs. 5 and 6 in Ch. 7).

10.6 We accept the following convention concerning entailment: Conditions $A, B \vdash C, D$ or $A, B \nvdash C, D$ mean respectively $A, B \vdash C$ and $A, B \vdash D$, $A, B \nvdash C$ and $A, B \nvdash D$; i.e. that $A$ and $B$ entails $C$ and that it entails also $D$, and that $A$ and $B$ does not entail $C$ and that it does not entail $D$.

Single cases $A \vdash B$ and $A \nvdash B$ are obtained by a suitable particularization.

10.7 Let's start with a consideration of the proper locative axioms: INL, EXL and $\mathbf{L}$. 
It is easy to see that each of them is weaker than the axioms of preorders (i.e. reflexivity and transitivity), as well as that the axioms of internal and external location are mutually independent, i.e., none of them entail the other:

\section{$\mathbf{P O} \vdash \mathbf{L}$ and $\mathbf{L} \nvdash \mathbf{P O}, \mathbf{E X L} \forall \mathbf{I N L}$ and $\mathbf{I N L} \not \forall \mathbf{E X L}$.}

As a matter of fact, the first three claims have been established already. Indeed, by (101) we knew that $\mathbf{P O} \vdash \mathbf{L}$ and by Ex. 3 of $\S 9.9$ we learnt that $\mathbf{L} \nvdash \mathbf{P O}$; in fact even more: $\mathbf{L} \nvdash \mathbf{R}, \mathbf{T}$.

On the other hand, by Ex. 1 of $\S 8.18$ we know that EXL, $\mathbf{T} \not \mathbf{I N L}, \mathbf{R}$.

For the last claim consider the following

Ex. 6 Let $U=\{x, y, z\}$ and $E=\Delta \cup\{x y, y z, z y\}$. Its diagram is as follows:

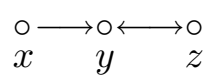

By a straightforward calculation we obtain that $P=\Delta \cup\{x y, z y\}, C=$ $\Delta \cup\{z y, y z\}, L=\Delta \cup\{x y, y z, z y\}$ and $A=\Delta \cup\{y z, z y\}$. Thus $L=E$, $P<E, A=C$. Hence $\langle U, E\rangle \models \mathbf{I N L}, \mathbf{A C}, \boldsymbol{P L}, \mathbf{R}$; whereas $\langle U, E\rangle$ falsifies EXL, L, T.

A fortiori, INL, $\mathbf{R} \nvdash \mathbf{E X L}, \mathbf{T}$.

10.8 Notice that, by (98), using the converses of the models generated by examples Ex. 1 and Ex. 6 we obtain

INL, $\mathbf{T} \nvdash \mathbf{E X L}, \mathbf{R}$ and $\mathbf{E X L}, \mathbf{R} \nvdash \mathbf{I N L}, \mathbf{T}$.

On the other hand observe that neither does location plus reflexivity imply transitivity nor does location plus transitivity imply reflexivity:

$$
\mathbf{L}, \mathbf{R} \nvdash \mathbf{T} ; \mathbf{L}, \mathbf{T} \nvdash \mathbf{R} .
$$

Proof. Let's start with the second claim: location plus transitivity does not imply reflexivity.

Consider the simplest case of a nonempty relation one can find:

Ex. $7 U:=\{x, y\}, E:=\{x y\}$, i.e.,

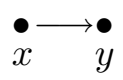

We immediately obtain that $P=\Delta \cup E, C=\Delta \cup E$ and $L=E=A$. Thus our model validates both transitivity (for $E \leqslant P$ ) and location but not reflexivity, as required. 
To confirm the first claim: location plus reflexivity does not imply transitivity, consider the following model:

Ex. $8 U:=\{1,2,3, \ldots\}, E:=\{n n: n \geqslant 1\} \cup\{\langle n, n+1\rangle: n \geqslant 1\}$. The diagram of this relation is quite familiar:

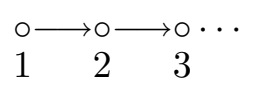

Let's calculate:

(i) $P=\Delta \cup\{\langle 1,2\rangle\}$.

Take first the case $n=1$. By definition of $P, 1 P m$ iff $\forall i(i E 1 \rightarrow i E m)$. But $i E 1$ iff $i=1$. Hence $1 P m$ iff $1 E m$ iff $m=1$ or $m=2$, as needed.

Next, consider the case $n \geqslant 2 . n P m$ iff for any $i, i E n \rightarrow i E m$. But $i E n$ iff $i=n-1$ or $i=n$. Hence $n-1 E m$ and $n E m$. Therefore $m=n$. I.e., for $n \geqslant 2, n P m$ iff $n=m$.

(ii) $C=\Delta$.

Let $n, m \geqslant 1$. By definition, $n C m$ iff for any $i, m E i \rightarrow n E i$. But $m E i$ iff $i=m$ or $i=m+1$. Therefore, $n C m$ iff $n E m$ and $n E m+1$ iff $n=m$.

(iii) $L=E$.

Let $n=1$. $1 L m$ iff for any $i, i P 1 \rightarrow i E m$. But $i P 1$ iff $i=1$. Hence $1 L m$ iff $1 E m$, as required.

Consider now the case $n \geqslant 2$. By (i), $i P n$ iff $i=n$. Therefore $n L m$ iff $n E m$, again as required.

(iv) $A=E$.

Immediately by (ii), for if $C=\Delta$ then $A=E$.

10.9 As a matter of fact, in the last two sections the four conditions: reflexivity $\mathbf{R}$, transitivity $\mathbf{T}$, internal location INL and external location EXL have been compared: For brevity's sake rename the last two of them by $\mathbf{I}$ and $\mathbf{E}$.

Connecting the above four relations in all logically different ways we obtain the following 15 formulas: T, R, I, E, TI (i.e. $\mathbf{T} \wedge \mathbf{I}$ ), TE, TR $(=\mathbf{P O}), \mathbf{I E}(=\mathbf{L}), \mathbf{R I}, \mathbf{R E}, \mathbf{T R E}, \mathbf{T I E}, \mathbf{R I E}$ and TRIE. What are the logical connections between these relations, i.e., which implications connect them?

Joining together (101), (104), (105) and (106) by means of the standard logical laws, we see that the diagram given below describes all implications between the four conditions under consideration: 


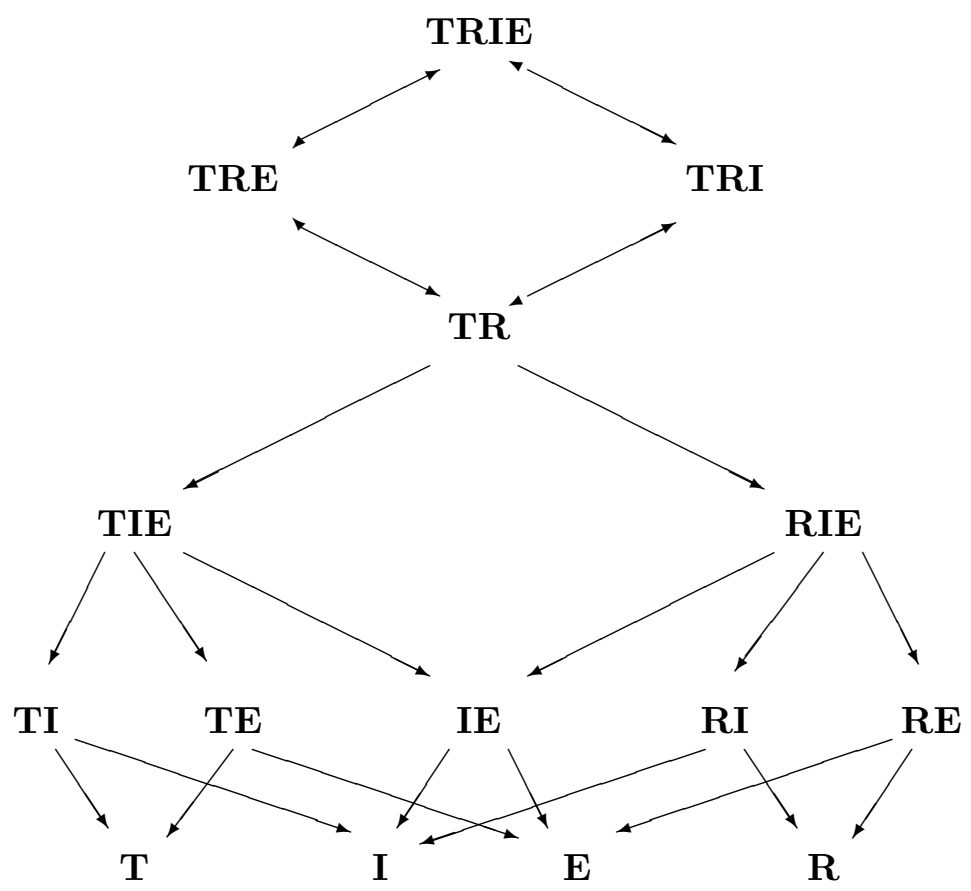

Fig. 8.

10.10 The model used in Ex. 8 calls into mind the realm of natural numbers. Let us discuss this matter more carefully.

The sequence of natural numbers is done by unlimited succession or infinite repetition. Usually, to describe this process the sequence function $s$ is introduced: $s(n):=n+1$. We start with 0 . Next we put: $1:=s(0)$, $2:=s(1)=s s(0), \ldots, n+1:=s(n), \ldots$.

The model of generation of natural numbers is depicted by the following diagram:

$\mathbf{N}$ :

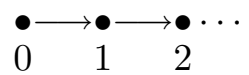

As a matter of fact, $\mathrm{N}$ models the function $s$. Its converse

$\underline{N}$ :

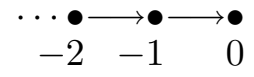

describes the function $s$ on the realm of negative integers, whereas their order-union:

$\mathbf{N}+\underline{\mathbf{N}}:$

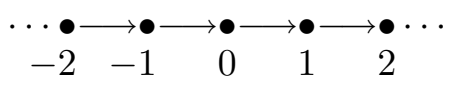


gives account of the basic structure of all integers.

Observe that none of the above models is transitive or reflexive. A fortiori, they are not preorders. Notice, that their preorder closure models the relation $\leqslant$ in each of the three respective domains.

Let me ask about the locative character of these basic arithmetical structures. By calculation similar to the one given in the second part of the proof of the last theorem we obtain

$$
\begin{aligned}
& \mathbf{N} \models \mathbf{E X L} \text { but } \mathbf{N} \not \models \mathbf{I N L}, \underline{\mathbf{N}} \models \mathbf{I N L} \text { but } \underline{\mathbf{N}} \not \models \mathbf{E X L} \text {, whereas } \\
& \underline{\mathbf{N}}+\mathbf{N} \models \mathbf{L} \text {. }
\end{aligned}
$$

In plain words: $\mathbf{N}$ is externally, but not internally locative; $\mathbf{N}$, conversely, is internally, but not externally locative, whereas the full structure of integers $\underline{\mathbf{N}}+\mathbf{N}$ is locative.

Thus, for a given $n$, the basic arithmetical function $s$ indicates its location: $n$ is located in $n+1$.

Finally notice, that the above succession models emerge by infinitization of the Russian doll's model, investigated in $\S 8.19$ and claimed there to be the natural, paradigmatic, model for location.

10.11 Compare now locative ontology and Leśniewski's ontology. We know (cf. Chapter 7) that Leśniewski's axiom implies transitivity but it does not imply reflexivity. Therefore

(108) $\quad \mathbf{L}, \mathbf{R} \nvdash \mathbf{L O N}$ : location plus reflexivity doesn't imply Leśniewski's axiom.

For otherwise $\mathbf{L}, \mathbf{R} \vdash \mathbf{T}$, which contradicts (106).

On the other hand,

(109) Leśniewski's axiom plus reflexivity does imply location: $\mathbf{L O N}, \mathbf{R} \vdash \mathbf{L}$.

For $\mathbf{L O N} \wedge \mathbf{R}$ is stronger even than preorders $\mathbf{P O}$.

By Ex. 7 of $\S 10.8$ we also know that location plus transitivity does not imply Leśniewski's axiom:

\section{$\mathbf{L}, \mathbf{T} \nvdash \mathbf{L O N}$}

for in the model of Ex. 7 we have $x E y$ but not $x E x$, contradicting one of the essential ingredients of Leśniewski's Ontology, cf. (42) of chapter 7. 
Also, Leśniewski's axiom does not imply location:

\section{LON $\nvdash \mathbf{L}$.}

To see this consider the following

Ex. $9 U:=\{x, y, z\}, E:=\{y y, y z\}$. Its diagram is as follows

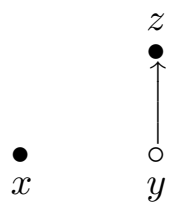

Clearly this model verifies $\mathbf{L O N}$ but falsifies $\mathbf{L}$, for $y$ is related to itself but not located in itself: $y E y$ but $\neg y L y$.

Finally, ask whether location plus Leśniewski's axiom imply reflexivity? The answer again is in negative:

\section{$\mathbf{L}, \mathbf{L O N} \nvdash \mathbf{R}$}

To see this it suffices to realize that the model containing only one simple item: $U=\{x\}, E=\emptyset$ is both locative and Leśniewskian, but not reflexive.

In conclusion, locative ontologies are new also in comparison with Leśniewski's structures.

10.12 In the last five sections quite a lot was calculated. A rather detailed description of the logical interconnections between the seven concepts: the four traditional - transitivity, reflexivity, preordering and Leśniewski's condition, and the three introduced here - internal location, external location and location has been presented.

For brevity's sake the notation introduced by the convention B is simplified here in the two basic cases: we write IS and ES instead of respectively INLS and EXLS, i.e., they respectively denote the class of all internally locative and the class of all externally locative structures.

The content of the last five sections can now be summarized by:

$$
\begin{array}{ll}
\text { i) } & \mathbf{T S} \cap \mathbf{R S}=\mathbf{P O S} \mp \mathbf{L S}=\mathbf{I S} \cap \mathbf{E S} \\
\text { ii) } & \mathbf{L S} \mp \mathbf{I S}, \mathbf{E S} \\
\text { iii) } & \mathbf{R S} \cap \mathbf{L S}-\mathbf{P O S} \neq \emptyset, \mathbf{T S} \cap \mathbf{L S}-\mathbf{P O S} \neq \emptyset \\
\text { iv) } & \mathbf{L S}-\mathbf{T S} \neq \emptyset, \mathbf{T S}-\mathbf{L S} \neq \emptyset, \mathbf{L S}-\mathbf{R S} \neq \emptyset \text { and } \mathbf{R S}-\mathbf{L S} \neq \emptyset \\
\text { v) } & \mathbf{L O N S} \mp \mathbf{T S}, \mathbf{P O S} \cap \mathbf{L O N S} \mp \mathbf{L S} \cap \mathbf{L O N S}
\end{array}
$$




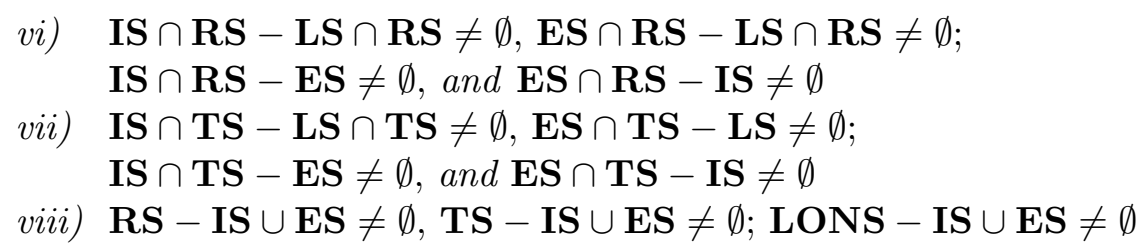

The above interrelations are pictured in the following Ontologische Salzburgerkugel:

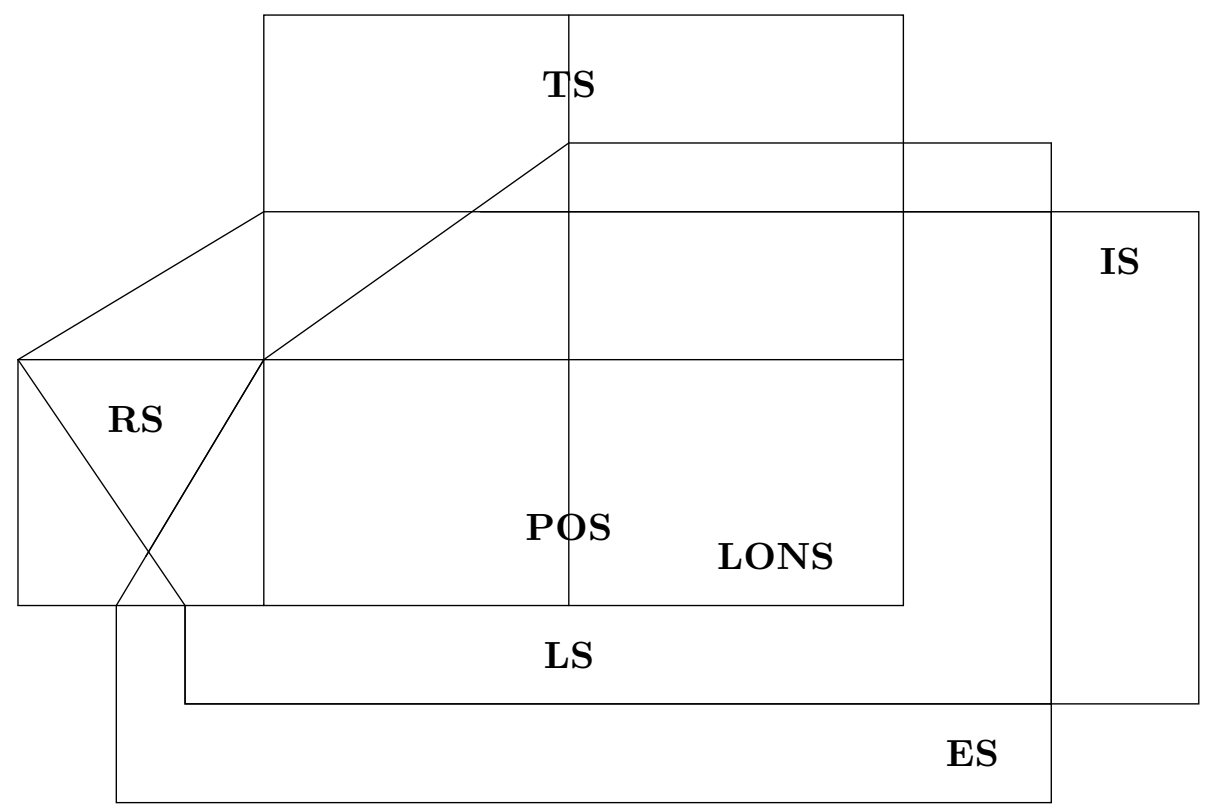

Fig. 9.

\section{Comparison Continued}

10.13 Turn now to domains satisfying axioms from the second family. By (81)-(87) from the sections 9.27-9.29 we know

$$
\begin{aligned}
& \text { i) } \quad P L S=R S \cap C A S \\
& \text { ii) } \mathbf{T S} \nsubseteq P L \mathbf{S} \cap C A \mathbf{S} \\
& \text { iii) } \mathbf{P O S} \varsubsetneqq \mathbf{L P S} \varsubsetneqq \mathrm{RS} \text { and } \mathbf{P O S} \varsubsetneqq \mathrm{ACS} \varsubsetneqq \mathrm{RS}
\end{aligned}
$$

Therefore, locative domains of the second type, like the basic domains of the first type, extend preorders, but in not so deviant way, for usually they are reflexive. 
On the other hand, by (113i) and (114iii), structures from the first family are, in a sense, complementary to ones from the second family. Taken separately they extend preorders in two different directions, while taken together they approximate them:

$$
\begin{aligned}
& \text { i) } \quad \mathbf{L P S} \cap \mathbf{I S}=\mathbf{P O S}=\mathbf{A C S} \cap \mathbf{E S} \text {, a fortior } \\
& \text { ii) } \mathbf{L P S} \cap \mathbf{L S}=\mathbf{P O S}=\mathbf{A C S} \cap \mathbf{L S} \text {. }
\end{aligned}
$$

Using the terminology of $\S 9.11$ we can say that preorders are exactly the locative and prelocative, or the locative and preallocative, orders.

10.14 Notice that

(116) LPS and ACS intersect each other.

To see this cf. Ex. 6 of $\S 10.7$ and its converse.

(117) LPS and $\mathbf{L S}$, as well as ACS and $\mathbf{L S}$, intersect each other.

Indeed, by (115), both pairs have the same intersection, namely the family of all preorders POS.

To see that $\mathbf{L}$ does not imply either $\mathbf{L P}$ or $\mathbf{A C}$ use the infinite succession model of $\S 10.10$; whereas Ex. 6 , with its converse, can be used to check that $A=C$, respectively $L=P$, does not imply $L=E=A$

10.15 The above interconnections, simplified by considering only one sort of basic locative structures (we omit IS and ES), are pictured in the second Salzburgerkugel:

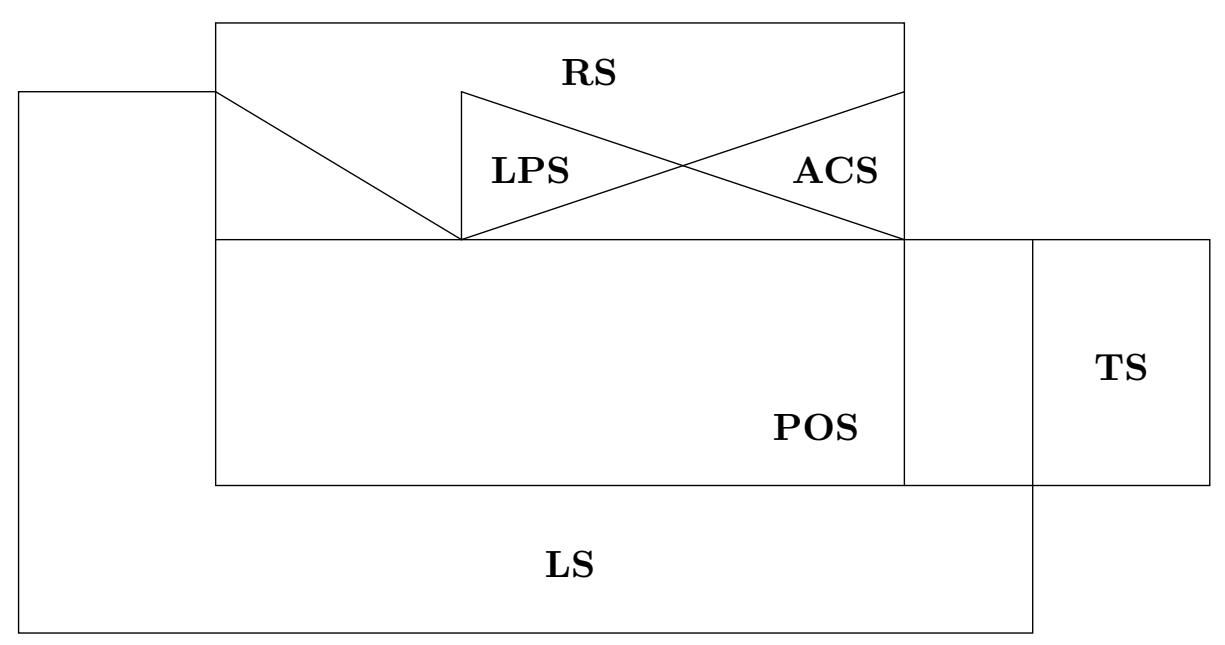

Fig. 10 . 
10.16 Finally, for the third group of locative structures, by the discussion of $\S 9.32$, we immediately obtain

(118) $\quad \boldsymbol{A} \boldsymbol{L} \mathbf{S} \cap \mathbf{I S} \varsubsetneqq \mathbf{E S}$ and $\boldsymbol{A} \boldsymbol{L S} \cap \mathbf{I S} \varsubsetneqq \mathbf{E S}$. Also $\mathbf{L S} \varsubsetneqq \boldsymbol{A} \boldsymbol{L S}$.

As a matter of fact

(119) $\quad \boldsymbol{A} \boldsymbol{L} \mathbf{S} \cap \mathbf{I S}=\mathbf{L S}=\boldsymbol{A} \boldsymbol{L} \mathbf{S} \cap \mathbf{E S}$

The above situation is depicted in the third Salzburgerkugel:

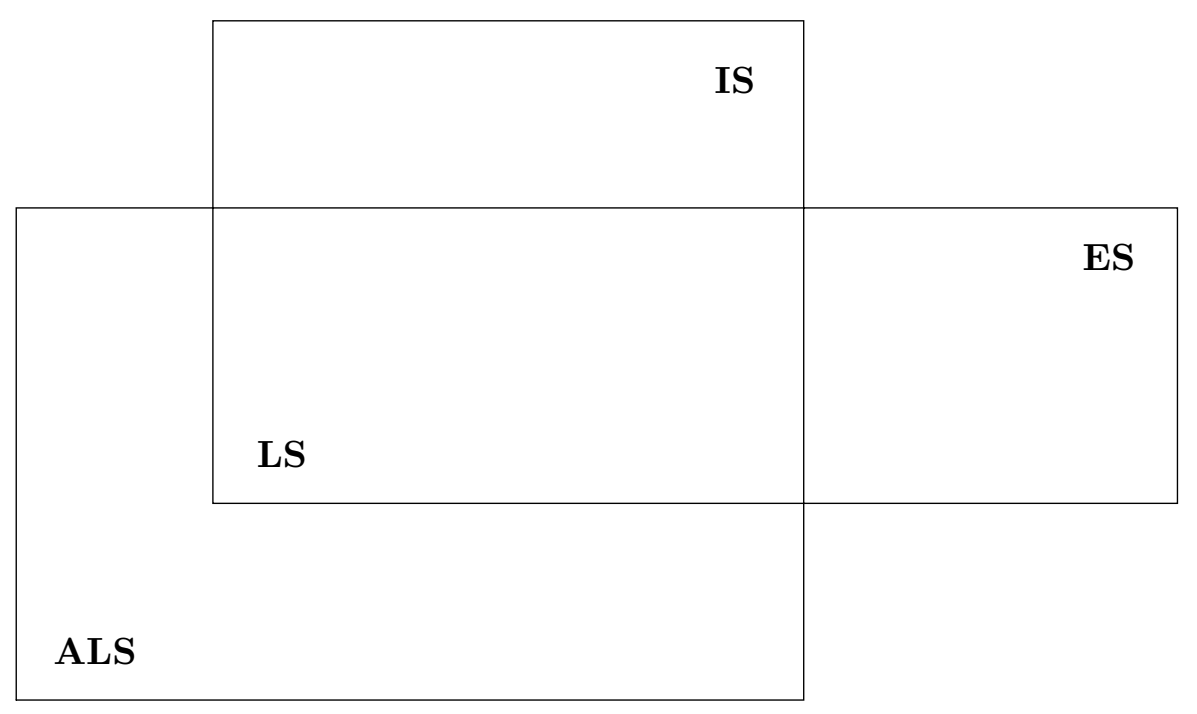

Fig. 11.

\section{Mereolocation}

10.17 In the previous sections of the present chapter our attention has been confined to comparison of preorders with locative structures of several types, i.e., to the investigation of the logical relations connecting axioms comparing the five relations involved: $E, P, C, L$ and $A$. Three cases appear to be particularly important: preorders in and of themselves - POS, and the two basic families of locative structures - IS and ES, i.e., axioms: $E=P, E=L$ and $E=A$, respectively.

Notice that until now we did not compare the mereological relation $M$ with location $L$. To complete our discussion I am going therefore to consider $M$ once again. 
10.18 Previously, in Chapter $6, M$ was compared with $E: E=M$, which defines premereologies. It was also compared there with $P: M=P$, which defines a bigger family of structures equal to premereologies in the realm of preorders, cf. (15) of $\S 6.6$.

Following our custom, PS and MPS are used to denote respectively the family of all premereologies (cf. 6.33) and the family of all relations satisfying MP: $M=P$.

By (15) we have

\section{$\mathbf{P S}=\mathbf{P O S} \cap \mathrm{MPS}$}

10.19 Observe that $M$, like $L, O$ and $P$ (cf. respectively (43), (23) and (2)), satisfies the following monotonicity law:

$$
z P x \wedge x M y \rightarrow z M y .
$$

Proof. Assume: i) $z P x$, i.e. $(z] \subseteq(x]$, ii) $x M y$, i.e. for any $u, u O x \rightarrow$ $u O y$, and iii) $u O z$, i.e. for some $w, w E u \wedge w E z$. By i), $w E x$. Hence $u O x$. Thus, by ii), $u O y$. Therefore $u O z \rightarrow u O y$, i.e. $z M y$, as required.

10.20 A metalogical conclusion is in order. The monotonicity of a given relation with respect to the parthood relation is too general, hence logically too weak, to characterize a given type of relation, for it fails to distinguish between $P, M, O$ and $L$.

10.21 The relation $E$ is said to be mereolocative iff its conjugate mereological and locative relations coincide:

ML

$$
M=L .
$$

The family of all mereolocative structures is denoted by MLS. Clearly, mereological containment is mereolocative:

(122) $\quad$ ME implies $\mathbf{M L}$, i.e., PS $\subseteq$ MLS.

Indeed, premereologies are preorders, which, in turn, are locative structures. Hence, $E=M$ and $E=L$, therefore $M=L$.

10.22 Is the reverse implication true? Does mereolocation coincide with mereological containment?

In what follows we will answer this question in the negative, thus clarifying that mereolocation is an essential weakening of the mereological relation. 
It does, however, preserve some important features both of location and of the mereological relation.

10.23 Notice that

(123) If $E$ is mereolocative, then $L$ is a preorder relation.

Indeed, by the assumption $L$ equals $M$ which, by (12), is known to be a preorder relation.

(124) If $E$ is mereolocative, then $E$ is reflexive, but not conversely:

$\mathbf{M L S} \varsubsetneqq \mathbf{R S}$.

Proof. By the previous claim, $L$ is reflexive, which, by (50), is equivalent to the reflexiveness of $E$. To see that the inclusion is proper take, for example, Ex. 7 of $\S 10.8$.

10.24 As a matter of fact, the axiom ML introduces a new class of structures which intersects each of the following three classes of structures: preorders, transitive structures and internally locative structures with the same trace - the class of all premereologies.

This fact is stated in the following sequence of claims:

(125) If $E$ is transitive and mereolocative, then $E$ is internally locative: $\mathbf{T S} \cap \mathbf{M L S} \subseteq \mathbf{I S}$.

Proof. By the first assumption and (18) we have $E \leqslant M$, whereas by the second one: $M=L$, hence $E \leqslant L$, which — by (69) — is equivalent to INL.

(126) Premereologies are exactly those relational structures which are both premerelogical and internally locative: $\mathbf{P S}=\mathbf{I S} \cap$ MLS.

Proof. By (13), (102) and (122) the right-hand inclusion is immediate.

For the reverse inclusion assume that $L=E$ and $M=L$. Hence $M=E$, as required.

Therefore, the axiom ML restricts the family of all internally locative structures to premereologies, as MP does in the realm of preorders.

(127) Premereologies are exactly those relational structures which are both premereological and transitive: $\mathbf{P S}=\mathbf{T S} \cap \mathbf{M L S}$. 
Proof. The right-hand inclusion follows again from (13) and (124).

For the left-hand inclusion apply (125): TS $\cap$ MLS $\subseteq$ IS. Hence TS $\cap$ MLS $\subseteq$ IS $\cap$ MLS. Using now (126) we obtain the required inclusion: TS $\cap$ MLS $\subseteq$ PS.

Analogously

(128) $\mathbf{P S}=\mathbf{M L S} \cap$ POS.

Observe that

(129) IS, TS and MLS cross each other. Also MLS and POS cross each other.

Proof. To prove the above claims we need a number of models. Consider first the following

$$
\begin{array}{cl}
\text { Ex. } 10 U:=\{i: i \geqslant 1\} & E:=\{j i: i, j \geqslant 1 \text { and } j=i \text { or } j=i+1\} \\
& \cdots \circ \longrightarrow \circ \longrightarrow \circ \\
3 & 2 \quad 1
\end{array}
$$

$E$ is reflexive, i.e. $\Delta \subseteq E$, but it is not transitive. We can easily check that $M=\Delta=P$, and $E=L$. Hence $M \varsubsetneqq L$. In conclusion: MP,INL $\forall \mathbf{M L}, \mathbf{T}$; hence both MPS and IS are included neither in MLS nor in TS.

Next, take

Ex. $11 U:=\left\{z, x, y, y_{1}, y_{2}\right\} \quad E:=\Delta \cup\left\{z x, x y, y_{1} y, y_{2} y\right\}$

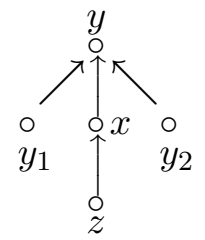

Here we can calculate that $P=\Delta \cup\left\{z x, y_{1} y, y_{2} y\right\}=L=M$. Hence $L<E$. $E$ again is reflexive, but not transitive.

In conclusion: $P=M, L=M \not \mathbf{I N L}$, T; hence both MPS and MLS are included neither in IS nor in TS.

In the next turn, consider the simplest model of all: a singleton with the empty relation. 
Ex. $12 U:=\{x\}, E:=\emptyset$.

Here $P=\{x x\}=M$, whereas $L=\emptyset=E$. Moreover, $E$ is transitive, but not reflexive. A fortiori, it is not a preorder relation.

Hence $\mathbf{T}, \mathbf{M P} \nvdash \mathbf{I N L}, \mathbf{M L}, \mathbf{R}, \mathbf{P O}$. Inter alia, TS $\not \subset \mathbf{I S}$ and TS $\nsubseteq \mathbf{M L S}$.

This finish the proof of the first claim.

To check that MLS and POS cross each other, notice firstly that MLS is not included in POS, because by Ex. 11 it, but not POS, is not included in TS; notice secondly that POS is not included in MLS, for otherwise $\mathbf{P S}=\mathbf{M L S} \cap \mathbf{I S}=\mathbf{P O S}$, which contradicts $\S 6.5$. QED

The connections calculated in the present section can be summarized in the following fourth ontological Salzburgerkugel:

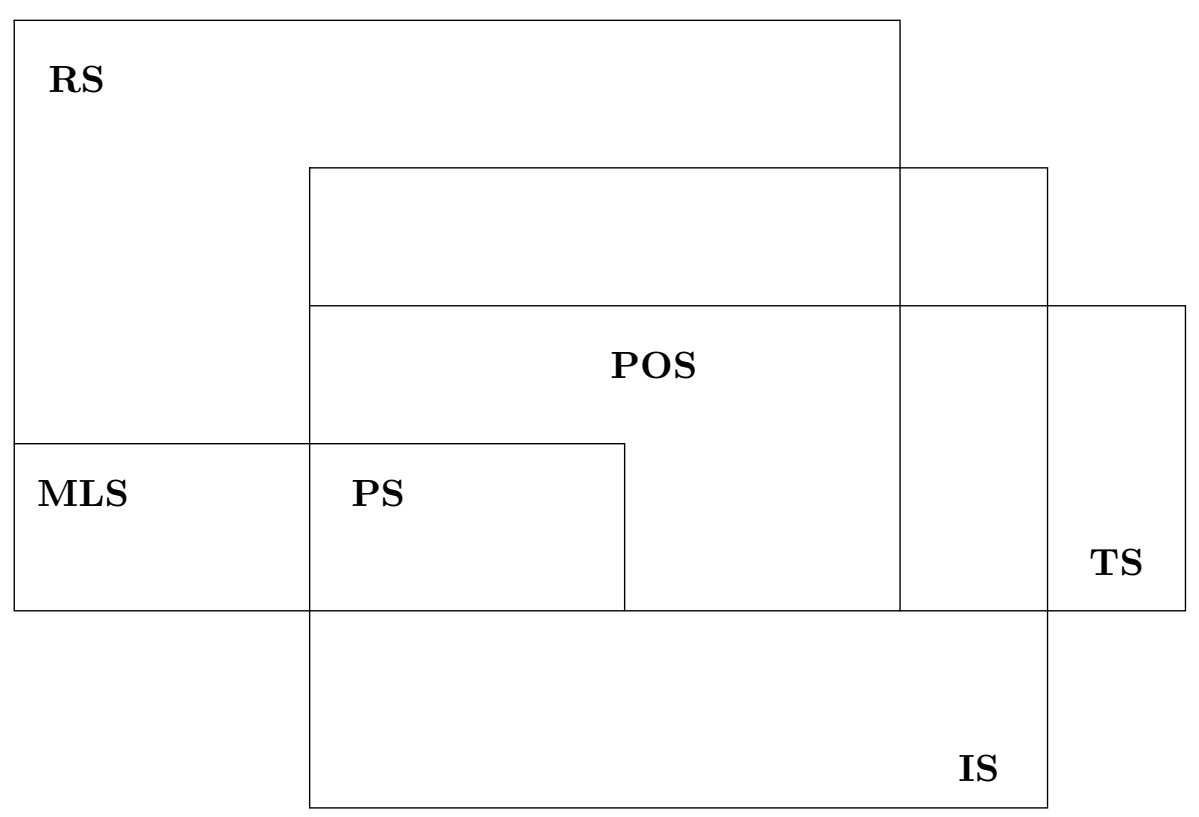

Fig. 12.

10.25 Turn now to the question of the interdependence between our twin-axioms:

ML $\quad M=L$ and

MP $M=P$. 
By the third model used in the proof of theorem (129), i.e. by Ex. 12, we know that

(130) $\mathbf{M P}$ does not imply $\mathbf{M L}$, even more: $\mathbf{M P}, \mathbf{T} \nvdash \mathbf{M L}$.

10.26 What is true of the reverse implication? Observe first that in general

(131) ML implies $P \leqslant M$.

Proof. Assume $L=M$ and $\neg(P \leqslant M)$. I.e., for some $x$ and $y, x P y$ and $\neg(x M y)$. Hence $\neg(x L y)$, i.e., for some $u: u P x$ and (i) $\neg(u E y)$. By $x P y$, $u P x$ and transitivity of $P$ we have $u P y$. Therefore, if $z E u$ then $z E y$. But by (124) $E$ is reflexive, hence $u E u$. Therefore $u E y$, which contradicts (i).

ML seems and really is a rather strong axiom. It implies, inter alia, that $L$ and $E$ are reflexive, hence $P \leqslant L \leqslant E$. On the other hand, as we just proved, it implies $P \leqslant M$.

However, it is too weak to imply the reverse inequality: $M \leqslant P$.

(132) ML does not imply MP.

Proof. Consider the following model:

Ex. $13 U:=\left\{x_{1}, x_{2}, x_{3}, x_{4}, x_{5}\right\}$

$$
E:=\Delta \cup\left\{x_{1} x_{3}, x_{1} x_{5}, x_{2} x_{3}, x_{3} x_{4}, x_{4} x_{3}, x_{4} x_{5}, x_{5} x_{4}, x_{5} x_{3}\right\}
$$

Its diagram is as follows:

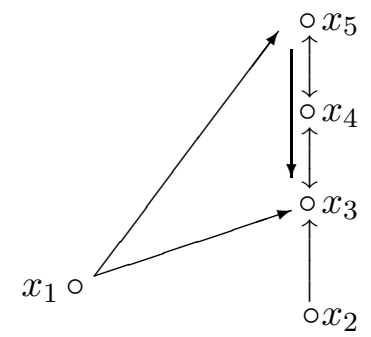

Now we can check that $P=\Delta \cup\left\{x_{1} x_{3}, x_{1} x_{5}, x_{2} x_{3}, x_{4} x_{3}\right\}, L=\Delta \cup$ $\left\{x_{1} x_{3}, x_{1} x_{5}, x_{2} x_{3}, x_{4} x_{3}, x_{4} x_{5}, x_{5} x_{3}\right\}=M$. Hence $\Delta<P<L=M<E$.

Therefore Ex. 13 verifies ML but falsifies $\mathbf{M P}$, a fortiori MP, as required. 
10.27 The information concerning MP which we collected in (15), (120), (130)-(132) enables us to complete the map of Fig. 12 by the following fifth Salzburgerkugel:

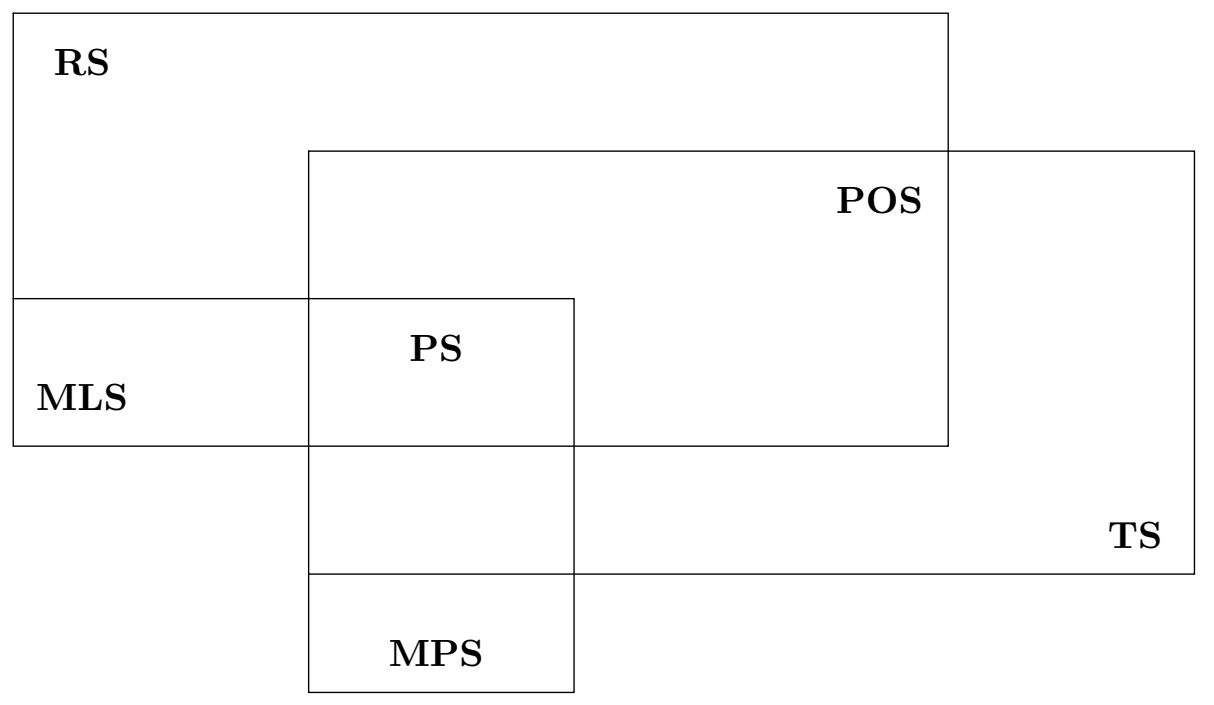

Fig. 13.

10.28 It is clear that the axiom of mereolocation implies the "condensing" part of the premereological axiom of condensation:

(133) $\quad M=L \rightarrow M \leqslant E$.

What we can say truly on the remaining part of ME? Call the suitable formula the axiom of semitransitivity:

$E M$

$$
E \leqslant M .
$$

The reason for this name is as follows: in the claim (18) of $\S 6.7$ it was stated that transitivity entails $E \leqslant M$.

On the other hand, it is easy to check that $\boldsymbol{E M}$ does not imply $\mathbf{T}$. To this end take the following intransitive model:

Ex. $14 U:=\{x, y\}, \quad E:=\{x y, y x, x x\}$

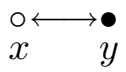

Here $P=\Delta \cup\{y x\}, L=\{y x\}$ and $O=U^{2}=M$. Therefore $E \leqslant M$, but $P$ crosses $E$, as required. 
In conclusion

(134) Each transitive frame is semitransitive, but not conversely: $\mathbf{T S} \varsubsetneqq \boldsymbol{E} M \mathbf{S}$.

We immediately obtain

(135) Each semitransitive and mereolocative frame is internally locative: EMS $\cap$ MLS $\subseteq$ IS.

Clearly

(136) Each premereology is both semitransitive and mereolocative: $\mathbf{P S} \subseteq \mathbf{E M S} \cap \mathbf{M L S}$ (126):

Using (135), (136) and (126) we obtain the following generalization of

(137) Premereologies are exactly these frames which are both semitransitive and mereolocative: $\mathbf{P S}=\boldsymbol{E} \mathbf{M S} \cap \mathbf{M L S}$.

Proof. We need only to check the left-hand inclusion, which, by (135), is immediate: $\boldsymbol{E M S} \cap \mathbf{M L S} \subseteq \mathbf{I S} \cap \mathbf{M L S}=\mathbf{P S}$.

10.29 Finally notice

(138) Semitransitive MP-frames are transitive, but not conversely: EMS $\cap \mathbf{M P S} \varsubsetneqq \mathbf{T S}$.

Proof. Assume $E \leqslant M=P$. Hence $E \leqslant P$ which, by (4), is known to characterize transitivity.

To prove the second claim take any preorder which is not premereological, for example: each nontrivial Boolean algebra. Recall (15): In the realm of preorders MP characterizes premereologies. Hence any Boolean algebra verifies $\mathbf{T}$ but falsifies $\mathbf{M P}$. Therefore $\mathbf{T}$ does not imply $\mathbf{M P}$, as was implicitely claimed.

10.30 And the last, but not least, move in the present search for reasonable modifications of a few well-established structures concerns the case of semimereolocation and the case of semiprelocation.

The relation $E$ is said to be semimereolocative iff it fulfils the following equation:

SML

$$
M \cap E=L ;
$$


whereas it is called semiprelocative iff the following equality holds:

SPL

$$
P \cap E=L .
$$

First of all

(139) If $E$ is mereolocative, then $E$ is semimereolocative, but not conversely: $\mathbf{M L S} \mp \mathbf{S M L S}$.

Proof. As regards the first claim assume $M=L$. We know that $L \leqslant E$, i.e., $L=L \cap E$. Hence $L=L \cap E=M \cap E$, as required.

For the second claim consider the following

Ex. $15 U:=\{x, y, z\}, \quad E:=\{x y, y z\}$

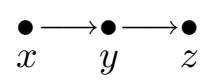

Here $P=\Delta \cup\{x y\}, L=\{x y\}$ and $M=\Delta \cup\{x y, x z\}$. Thus $M \cap E=L$, but $M \neq L$, as required. Observe that in our model $E, P$ and $M$ cross each other.

(140) If $E$ is prelocative, then $E$ is semiprelocative, but not conversely: PLS $\varsubsetneqq$ SPLS.

Proof. The argument for the first claim is, mutatis mutandis, the same as the argument for the analogous claim in the previous theorem.

To prove the second claim return to Ex. 7 of $\S 10.8$. There indeed $P \cap E=$ $L$, but $P \neq L$, as required.

10.31 To locate new structures with respects to preorders observe:

(141) If $E$ is a preorder (and thus, a fortiori, premereology), then $E$ is semimereolocative and semiprelocative. None of the above inclusions is reversible.

Proof. Assume that $E$ is a preorder relation, hence $E=P=L$. By (134), $E=E \cap M$. Therefore $L=E \cap M$.

The second inclusion immediately follows from (140).

To prove that semimereolocation does not imply transitivity, hence the axiom PO of preorders, use again Ex. 15; whereas the last claim can be checked by use of Ex. 7, where $P \cap E=L$, but $E$ is not reflexive, hence it falsifies $\mathbf{P O}$.

10.32 Confront now both types of frames just introduced with suitable versions of transitivity: 
$(142)$ i) If $E$ is semitransitive and semimereolocative, then $E$ is internally locative, but not conversely: $\mathbf{E M S} \cap \mathbf{S M L S} \mp \mathbf{I S}$

ii) If $E$ is transitive and semiprelocative, then it is internally locative, but not conversely: $\mathbf{T S} \cap \mathbf{S P L S} \nsubseteq \mathbf{I S}$

Proof. Assume $E \leqslant M$ and $M \cap E=L$. Hence $E=M \cap E=L$, as required.

The first claim of ii) is proved by the same, mutatis mutandis, argument.

For the second claims of both i) and ii) reconsider the model $\underline{\mathbf{N}}+\mathbf{N}$ of $\S 10.10$. There $E=L, E \cap \Delta=\emptyset$ and $M=\Delta=P$. Therefore $M \cap E=\emptyset=$ $P \cap E$, but both $L$ and $P$ are nonempty.

Notice that (142i) generalizes (135).

$(143)$ i) Internally locative and semimereolocative frames are semitransitive, but not conversely: IS $\cap \mathbf{S M L S} \varsubsetneqq \boldsymbol{E} \mathbf{M S}$

ii) Internally locative and semiprelocative frames are transitive, but not conversely: $\mathbf{I S} \cap \mathbf{S P L S} \varsubsetneqq \mathbf{T S}$

Proof. Ad i) Assume $L=M$ and $M \cap E=L$. Then $M \cap E=E$, i.e., $E \leqslant M$, as required.

To check the second claim consider, for example, Ex. 14 of $\S 10.28$ where indeed $L<E<M$, hence ST does not imply INL.

As immediate corollaries we have

$(144)$ i) Semimereolocative and semitransitive frames are exactly semimereolocative and internally locative frames:

$$
\text { EMS } \cap \mathbf{S M L S}=\mathbf{I S} \cap \mathbf{S M L S}
$$

ii) Frames which are semiprelocative and transitive are exactly semiprelocative and internally locative ones:

\section{$\mathbf{T S} \cap \mathbf{S P L S}=\mathbf{I S} \cap \mathbf{S P L S}$}

Proof. Ad i) By (142i) $\boldsymbol{E M S} \cap \mathbf{S M L S} \subseteq$ IS $\cap \mathbf{S M L S}$, whereas by (143i) $\mathbf{I S} \cap \mathbf{S M L S} \subseteq \boldsymbol{E} \boldsymbol{M S} \cap \mathbf{S M L S}$, hence both intersections coincide: $\mathbf{I S} \cap \mathbf{S M L S}=E M \mathbf{S} \cap \mathbf{S M L S}$.

To prove the claim ii) use a similar combination of (142ii) and (143ii).

10.33 In conclusion: Transitivity and semitransitivity play quite similar, interchangable, role in combination respectively with semiprelocation and semimereolocation, in both cases indicating internally locative structures. 
As regards appropriate intersections, they clearly contain preorders:

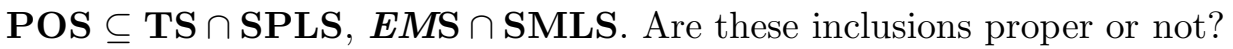

Yes, they are. As regards the second inclusion: POS $\subsetneq \boldsymbol{E} \boldsymbol{M} \mathbf{S} \cap \mathbf{S M L S}$ use Ex. 12 of $\S 10.24$, whereas the properness of the first inclusion: POS $\varsubsetneqq \mathbf{T S} \cap$ SPLS follows by checking the following

Ex. $16 U:=\{x, y, z\}, E:=\{x y, x z, y z\}$

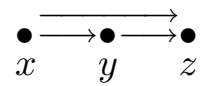

Here $L=E$ and $P=\Delta \cup E$, hence $E=L<P$, as needed.

10.34 The above connections concerning semimereolocation lead to the following sixth Salzburgerkugel, being a rafined version of Fig. 12 (its dashed part corresponds to $\mathbf{I S} \cap \mathbf{S M L S}=\mathbf{I S} \cap \boldsymbol{E M} \mathbf{S})$ :

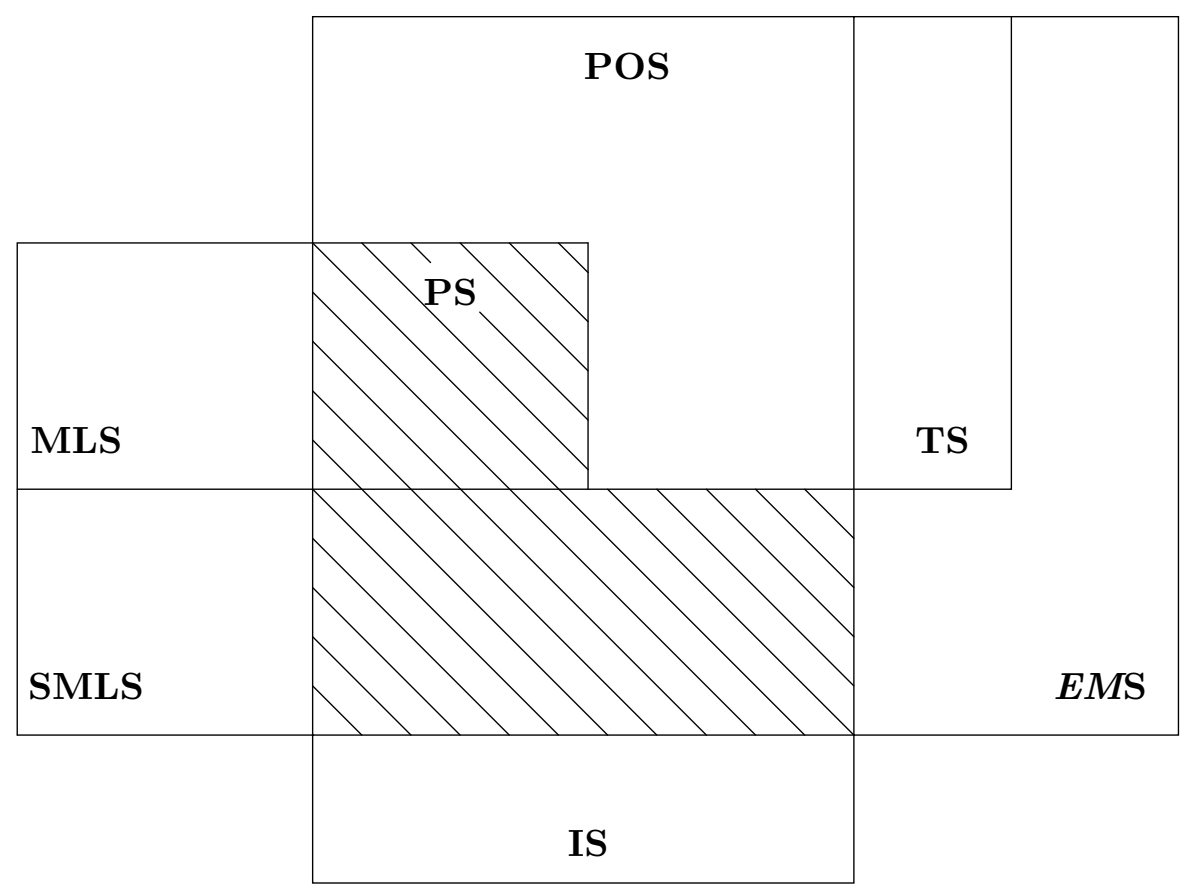

Fig. 14 .

An analogous diagram can be drawn to indicate interrelations which characterize prelocations. 


\section{Conclusion}

10.35 The complex picture of all connections pointed out in the present chapter can now be obtained by joining together all six Salzbergerkugeln. This is left to the reader's imagination, because the picture is too complex for the rather restricted drawing abilities of the present author.

10.36 Semitransitivity indeed seems to be a reasonable generalization of transitivity. This suggests a suitable generalization of the notion of preorder.

Let a reflexive and semitransitive relation $E$ be called a semipreorder relation. The family of all semipreorders is denoted by SPOS. Clearly

(145) $E$ is a semipreorder relation iff $P \leqslant E \leqslant M$.

The place of semipreorders with respect to other kinds of frames can easily be delineated by analysis of the previous diagrams. Inter alia

(146) i) Semimereolocative semipreorders are internally locative, but not conversely: SPOS $\cap \mathbf{S M L S} \subsetneq \mathbf{I S}$;

ii) Semiprelocative semipreorders are prelocative, but not conversely: SPOS $\cap \mathbf{S P L S} \nsubseteq \mathbf{P L S}$.

10.37 The variety of reasonable types of relational frames is both amazing and amusing. In this chapter we touch only a small, but important part of it. What we saw, however, calls for further research and attention.

\section{(To be continued)}




\section{Acknowledgments}

The main body of locative ontology was calculated in April 1990 while I was a guest of the Institute of Philosophy, Salzburg University. I wish to thank here the members of the Institute who helped to make my stay both a pleasant and a profitable one, in particular Prof. Paul Weingartner for the invitation, to Prof. Peter M. Simons for helpful discussions, and to Maria Fellner, Alexander Hieke and Maria Maier for their supporting interest in my lectures. The results were presented also at the XXXVI-th Kraków Logical Conference, November 13, 1990.

The second, much more extended version of the paper was written during my stay in the Internationale Akademie für Philosophie im Fürstentum Liechtenstein as research fellow co-working on the project "Formal Ontology and the Philosophical Foundations of Artificial Intelligence", led by Prof. Barry Smith under the auspices of Swiss National Foundation for Scientific Research. Thanks go both to the SNF and the IAP for their support and hospitality. Thanks are also due to R. Casati for his comments and to B. Smith for helpful and inspiring discussions.

Support of the KBN grant no. 1 P101 00205 is also thankfully acknowledged. 


\section{References}

[1] J. van Benthem, Essays in Logical Semantics, D. Reidel Publ. Co., Dordrecht 1986.

[2] A. Brückner, Stownik etymologiczny języka polskiego, Wiedza Powszechna, Warszawa 1970.

[3] P. M. Cohn, Universal Algebra, D. Reidel Publ. Co., Dordrecht 1981.

[4] F. Drake, Set Theory, N. Holland Publ. Co., Amsterdam 1974.

[5] Ch. Kahn, The Verb "Be" in Ancient Greek, D. Reidel Publ. Co., Dordrecht 1973.

[6] A. Krạpiec, Metafizyka, Tow. Naukowe KUL, Lublin 1978.

[7] S. Leśniewski, Podstawy ogólnej teorii mnogości, Moskwa 1916.

[8] S. Leśniewski, "O podstawach matematyki", Przeglad Filozoficzny, XXX (1927), p. 164-206; XXXI (1928), p. 261-291; XXXII (1929), p. 60-101; XXXIII (1930), p. 77-105; XXXIV (1931), p. 142-170.

[9] S. Leśniewski, Collected Works, vols. I\&II, ed. by S. J. Surma, J. T. Srzednicki, D. I. Burnett and V. F. Rickey, Kluwer Acad. Publ., Dordrecht 1992.

[10] M. Libardi, Teorie delle parti e dell'interno. Mereologie estensionali, Centro Studi Per La Filosofia Mitteleuropea, Quaderni II.1-3, Trento 1990.

[11] E. Mendelson, Introduction to Mathematical Logic, 2nd ed., D. van Nostrand Co., New York 1979.

[12] J. Perzanowski, "Byt", Studia Filozofuczne, 1988, nr 6/7 (271/72), p. 63-85.

[13] J. Perzanowski, "Ontologies and Ontologics", in: Logic Counts, ed. by E. Żarnecka-Biały, Kluwer Academic Publishers, Dordrecht 1990, p. 23-42.

[14] J. Perzanowski, Logiki modalne a filozofia, Wyd. UJ, Kraków 1989.

[15] J. Perzanowski, "The Way of Truth", in: Formal Ontology, ed. by R. Poli and P. Simons, Kluwer Academic Publishers.

[16] J. Perzanowski, "A Theory of Qualities and Substance I: The Ontological Theorem" (in preparation).

[17] J. Perzanowski, "A Theory of Qualities and Substance II: Elements of Elementologic" (in preparation).

[18] J. Perzanowski, "Nominalism of Leśniewski's Ontology" (in preparation).

[19] J. Perzanowski, "Modalities, Ontological", in: Handbook of Metaphysics and Ontology, ed. by H. Burkhardt and B. Smith, Philosophia Vlg., München 1991. 
[20] J. Perzanowski, "Combination Ontology I: Analysis and Synthesis" (in preparation).

[21] J. Perzanowski, "Combination Ontology II: Ontological Modalities" (in preparation).

[22] J. Perzanowski, "Combination Ontology III: Combination Semantics" (in preparation).

[23] J. Perzanowski, Badania Onto-logiczne (work in progress).

[24] E. Schröder, Vorlesungen über die Algebra der Logik (Exakte Logik), Teubner, Leipzig, 1890-1910. Reprinted Chelsea, New York, 1966.

[25] P. Simons, Parts. A Study in Ontology., Clerendon Press, 1987.

[26] B. Smith, "On the Phases of Reism", in: Kotarbinski: Logic, Semantics and Ontology, ed. by J. Woleński, Kluwer Academic Publishers, Dordrecht 1990, p. $137-183$.

[27] J. T. Srzednicki and V. F. Rickey (eds.): Leśniewski's Sytems: Ontology and Mereology, Nijhoff, The Hague, 1984.

[28] A. Tarski, "On the Calculus of Relations", Journal of Symbolic Logic, 6(1941), p. $73-89$.

[29] D. Westerståhl, "Quantifiers in Formal and Natural Languages", in: D. Gabbay and F. Guenthner (eds.) Handbook of Philosophical Logic, vol. IV, D. Reidel Publ. Co., Dordrecht 1989, p. 1-131.

\author{
JERZY PERZANOWSKI \\ Department of Logic \\ N. Copernicus University \\ 87-100 Toruń, POLAND \\ Department of Logic \\ Jagiellonian University \\ Grodzka 52 \\ 31-044 Kraków, POLAND
}

